\title{
SUMMARY OF QUANTITATIVE INTERPRETATION OF IMAGE FAR ULTRAVIOLET AURORAL DATA
}

\author{
H.U. FREY ${ }^{1}$ (hfrey@ssl berkeley.edu), S.B. MENDE ${ }^{1}$, T.J. IMMEL ${ }^{1}$, J.-C. \\ GÉRARD $^{2}$, B. HUBERT ${ }^{2}$, S. HABRAKEN ${ }^{3}$, J. SPANN ${ }^{4}$, G.R. GLADSTONE ${ }^{5}$, D.V. \\ BISIKALO $^{6}$ and V.I. SHEMATOVICH ${ }^{6}$ \\ ${ }^{1}$ Space Sciences Laboratory, University of California Berkeley, Berkeley, CA 94720, USA \\ ${ }^{2}$ University of Liège, B-4000 Liège, Belgium \\ ${ }^{3}$ Centre Spatial de Liège, B-4031 Liège, Belgium \\ ${ }^{4}$ NASA George C. Marshall Space Flight Center, Huntsville, AL 35812, USA \\ ${ }^{5}$ Southwest Research Institute, San Antonio, TX 78228, USA \\ ${ }^{6}$ Institute of Astronomy, Russian Academy of Sciences, Moscow, Russia
}

\begin{abstract}
Direct imaging of the magnetosphere by instruments on the IMAGE spacecraft is supplemented by simultaneous observations of the global aurora in three far ultraviolet (FUV) wavelength bands. The purpose of the multi-wavelength imaging is to study the global auroral particle and energy input from the magnetosphere into the atmosphere. This paper describes the method for quantitative interpretation of FUV measurements. The Wide-Band Imaging Camera (WIC) provides broad band ultraviolet images of the aurora with maximum spatial resolution by imaging the nitrogen lines and bands between 140 and $180 \mathrm{~nm}$ wavelength. The Spectrographic Imager (SI), a dual wavelength monochromatic instrument, images both Doppler-shifted Lyman- $\alpha$ emissions produced by precipitating protons, in the SI-12 channel and OI $135.6 \mathrm{~nm}$ emissions in the SI-13 channel. From the SI-12 Doppler shifted Lyman- $\alpha$ images it is possible to obtain the precipitating proton flux provided assumptions are made regarding the mean energy of the protons. Knowledge of the proton (flux and energy) component allows the calculation of the contribution produced by protons in the WIC and SI-13 instruments. Comparison of the corrected WIC and SI-13 signals provides a measure of the electron mean energy, which can then be used to determine the electron energy flux. To accomplish this, reliable emission modeling and instrument calibrations are required. In-flight calibration using early-type stars was used to validate the pre-flight laboratory calibrations and determine long-term trends in sensitivity. In general, very reasonable agreement is found between in-situ measurements and remote quantitative determinations.
\end{abstract}

Keywords: IMAGE, Far Ultraviolet instrument, FUV, aurora, quantitative, proton, electron, flux and energy

\section{Introduction}

Previously flown satellite imaging experiments have demonstrated the suitability of the vacuum ultraviolet spectral region for remote sensing observation of auroral precipitation (Frank et al., 1981; Anger et al., 1987; Frank and Craven, 1988; Murphree et al., 1994). In the wavelength region 120-190 nm, a downwardviewing imager is minimally contaminated by scattered sun light from clouds and the ground and the radiance of the aurora observed in a nadir viewing geometry can 
be distinguished from the high latitude dayglow. The UV emissions thus permit quantitative imaging of the auroral regions (Lummerzheim et al., 1997). An instrument for such observations should have adequate wavelength resolution to separate key spectral features, e.g. high enough spectral resolution to distinguish Lyman- $\alpha$ produced by proton precipitation from the geocorona. The auroral Lyman- $\alpha$ line provides a measure of the proton flux precipitating into the atmosphere.

The IMAGE satellite far ultraviolet (FUV) imaging system was built with these requirements in mind. It consists of the Wideband Imaging Camera (WIC) and the Spectrographic Imager (SI). The WIC provides broad band ultraviolet images of the aurora for maximum spatial resolution. The Spectrographic Imager (SI) makes quantitative images of different types of aurora, filtering them by wavelength.

The subsolar dayglow intensity in the FUV region from 100-170 nm, is less than 100 Rayleigh/nm when viewed in nadir (Meier, 1991). The dayglow continuum brightens by 2 orders of magnitude at wavelengths higher than $170 \mathrm{~nm}$, so it is particularly important to suppress emissions at longer wavelengths. There are strong line emissions at Lyman- $\alpha, 130.4$ and $135.6 \mathrm{~nm}$ from the dayglow. Fortunately most auroral imaging of the polar atmosphere takes place with the solar illumination at a large slant angle and the dayglow contribution is therefore much reduced compared to equatorial regions.

The Lyman- $\alpha$ emission near $121.6 \mathrm{~nm}$ has two components: the very intense cold Lyman- $\alpha$ of the geocorona centered precisely at $121.567 \mathrm{~nm}$ and the weaker Doppler-shifted auroral hydrogen emission produced as precipitating protons interact with the ambient atmosphere. There is a high probability for protons that charge exchange within the atmosphere to become neutral in an excited state of the $\mathrm{H}$ atom which will decay to the ground state through the Lyman- $\alpha$ transition. The resulting radiation will be Doppler-shifted to longer wavelength because the motion of the precipitating $\mathrm{H}^{+} / \mathrm{H}$ is primarily away from an observing satellite (Lummerzheim and Galand, 2001). The IMAGE FUV Spectrographic imager SI-12 channel was designed and built to observe these Doppler-shifted Lyman- $\alpha$ emissions.

Although the brightest FUV line in the aurora is the $130.4 \mathrm{~nm}$ OI emission, it is strongly scattered in the atmosphere and it is difficult to obtain an intensity distribution of the nascent auroral source. The $135.6 \mathrm{~nm}$ line is scattered to a lesser degree and provides an excellent emission feature for imaging the aurora (Strickland and Anderson, 1983). Thus, an important measurement requirement is the detection and spectral separation of the $135.6 \mathrm{~nm}$ emission from that at $130.4 \mathrm{~nm}$. The transmission of the instrument at $130.4 \mathrm{~nm}$ should be less than $1 \%$ of its $135.6 \mathrm{~nm}$ transmission. For IMAGE this necessitated the use of a spectrometer because the state of the art in FUV narrow band filter technology could not satisfy this requirement. The SI-13 wavelength channel images this emission feature.

The FUV Lyman-Birge-Hopfield (LBH) system and a few lines of atomic nitrogen populate the rest of the FUV region, which are produced primarily by electron impact excitation of $\mathrm{N}_{2}$. A method for obtaining precipitating electron energy parameters from FUV observations is proposed by (Strickland et al., 1983; Strickland 
et al., 1993). Atmospheric $\mathrm{O}_{2}$ is an absorbing agent residing mostly below $120 \mathrm{~km}$, and emissions that come from deeper in the atmosphere tend to get absorbed by it in the mid FUV wavelength region. $\mathrm{O}_{2}$ absorption becomes less significant in the longer (>160 nm) wavelength range.

Thus, comparison of the emission intensities at lower and higher wavelengths within the FUV region yields a parameter that can be related to the altitude of emission, and taking it one step further, to the energy of the precipitating electrons (Germany et al., 1997). By inverting the relationship between the ratios of the emissions it is possible to obtain the mean energy and flux of the precipitating electrons. Using this method FUV emissions are most effective in discriminating particle energies in the region 1-15 keV. Electrons in the energy range below $1 \mathrm{keV}$ do not penetrate the $\mathrm{O}_{2}$ layer and there is little sensitivity to energy. Above $15 \mathrm{keV}$ the bulk of the emissions are strongly absorbed leaving only a small fraction of the intensity coming from high altitudes and thus likewise inhibiting the discrimination of energies. It was decided early in the planning of the IMAGE mission that we would have only one broad band LBH imager to image the auroral LBH, and not use the technique of comparing high and low wavelength LBH emissions.

Instead we model the OI $135.6 \mathrm{~nm}$ and $\mathrm{N}_{2}$ LBH system intensities (line-ofsight integral) with and without atmospheric absorption and compare the measured ratios with these theoretical predictions. It is then possible to infer the altitude of the precipitation, hence obtaining the energy of the precipitating electrons. The LBH and $135.6 \mathrm{~nm}$ OI emissions are produced by electron impact on $\mathrm{N}_{2}$ and $\mathrm{O}$, respectively. Therefore, energy estimates of the precipitating electrons based on their emission ratios have to rely on the assumption that the $\mathrm{O} / \mathrm{N}_{2}$ ratio of the atmosphere is known. This ratio, however, is variable and depends on magnetospheric activity (Strickland et al., 1999; Drob et al., 1999). In the absence of the two-channel LBH measurement it is necessary to make an assumption regarding the $\mathrm{O} / \mathrm{N}_{2}$ ratio. For example a model atmosphere can be used and the auroral mean energy and energy flux from the emission intensities can be inferred (Strickland et al., 1983).

In summary, the auroral FUV instrument on the IMAGE spacecraft is designed to make quantitative remote sensing measurements of global particle precipitation properties. In this paper we review the imaging properties of the IMAGE FUV experiment, discuss the in-flight calibrations performed since the launch of IMAGE, summarize the calibration parameters and show how these parameters can be applied to obtain precipitating particle properties.

\section{Discussion of Imaging Concepts}

In general, an imager is a remote sensing instrument that makes multiplexed measurements of photon fluxes simultaneously from several distinct directions. The optics of an imaging system usually consists of a single aperture collecting light from several directions and an optical system that sorts the photons into appro- 
priate pixels, according to the direction of arrival. To first order, imagers can be characterized by the following general considerations:

1. the ability to determine the mean direction of light entering a pixel

2. the spatial size of the region associated with a pixel

3. the efficiency of counting the photons within the pixel

4. the acceptance or rejection of photons based on their wavelength

The efficiency of an imager is characterized by measuring the number of photons, which contribute to the signal associated with a pixel. When a known number of photons $\mathrm{P} / \mathrm{cm}^{2}$ arrive at the input aperture during the exposure time the output signal to noise ratio (SNR) is measured. From the output signal-to-noise ratio it is possible to calculate the output signal $\mathrm{Q}$ ( $\mathrm{AD}$ units), which would produce the same signal-to-noise ratio through Poisson statistics i.e. $(\mathrm{SNR})^{2}=\mathrm{Q}$. The ratio of the output signal to the input photons, $\mathrm{Q} / \mathrm{P}$ is the efficiency of the imager. The product of the efficiency of the detector and the clear unobstructed area of the collecting aperture is the equivalent or effective collecting area $\mathrm{A}_{e}$ of an imager.

The surface brightness unit, Rayleigh, is used most often in the measurement of terrestrial aurora because it is independent from the distance between the emitting source and the observer. A Rayleigh is equivalent to a source strength in which a $1 \mathrm{~cm}^{2}$ area integrated line of sight column of aurora emits $10^{6}$ photons s $\mathrm{s}^{-1}$ in $4 \pi \mathrm{sr}$ (Hunten et al., 1956). Thus, the intensity of a Rayleigh is $10^{6} / 4 \pi \approx 80,000$ photons $\mathrm{s}^{-1} \mathrm{~cm}^{-2} \mathrm{sr}^{-1}$. To express the response of an imager in terms of equivalent output counts $\mathrm{Q} /$ Rayleigh, one needs to multiply the field of view in steradian, the exposure duration in seconds and the equivalent collecting area $\mathrm{A}_{e}$ by 80,000 .

$$
Q=\Omega A_{e} T_{\exp } 10^{6} / 4 \pi
$$

The FUV auroral instrument complement provides global images of the aurora to allow comparisons of auroral data to the magnetospheric IMAGE data. The location and shape of the auroral regions are important indicators of magnetospheric conditions providing important context information for the other IMAGE measurements. It is, therefore, a primary scientific requirement of IMAGE-FUV to cover the entire auroral oval from apogee. The Earth subtends a total of about $16^{\circ}$ from IMAGE apogee and the auroral oval is about $8^{\circ}$. Thus, a primary requirement of IMAGE-FUV is global coverage of the auroral oval from the apogee altitude of $45,000 \mathrm{~km}$. In the IMAGE FUV design, the WIC camera and the SI cameras have $17.2^{\circ} \times 17.2^{\circ}$ and $16.3^{\circ} \times 16.3^{\circ}$ field of views, respectively.

The required spatial resolution can be obtained by considering that it is desirable to see features that are on the order of $100 \mathrm{~km}$ in size. To satisfy this requirement, the WIC design has $256 \times 256$ pixels and SI has $128 \times 128$ pixels. The projection or foot print of such a nominal pixel at $7 \mathrm{R}_{E}$ distance is $100 \times 100 \mathrm{~km}^{2}$ for the SI and $52 \times 52 \mathrm{~km}^{2}$ for the WIC. For $1000-\mathrm{km}$ perigee observations from IMAGE orbit the nominal pixel sizes are $1.2 \times 1.2$ and $2.2 \times 2.2 \mathrm{~km}^{2}$ for the WIC and SI, respectively. 
Once the pixel size is defined we can calculate the solid angle subtended by each pixel, $\left(1.37 \times 10^{-6}\right.$ sr and $4.9 \times 10^{-6}$ sr, for WIC and SI). As discussed previously, the photon arrival rate, $\mathrm{P}_{i}$ per Rayleigh of any imager viewing with a pixel solid angle of $1.37 \times 10^{-6} \mathrm{sr}$ is 0.11 photons s ${ }^{-1}$ Rayleigh $^{-1}$ for each $\mathrm{cm}^{2}$ of input aperture. Similarly for a SI pixel, the photon arrival rate, $\mathrm{P}_{i}$ is 0.39 photons $\mathrm{s}^{-1}$ Rayleigh $^{-1} \mathrm{~cm}^{-2}$. It is clear that these calculated photon arrival rates are independent of the instrument and are only defined by the geometry of the observation dictated by the desired resolution. The instrument intercepts this photon flux and produces a signal during an exposure time of $\mathrm{T}_{\text {exp }}$ seconds.

The instrument equivalent aperture $\mathrm{A}_{e}$ usually depends on the wavelength. The wavelength response of $\mathrm{A}_{e}$ was calibrated on the ground for all instrument channels by applying known monochromatic UV beams. These data are necessary to interpret the response of the system to any broad band light source whether the source is auroral or stellar. All in-flight calibrations, therefore, depend on these relative wavelength calibrations performed in the lab.

The sensitivity in terms of counts per Rayleigh also depends on the spectral distribution of the input radiation. Nevertheless it is possible to calibrate the instrument and obtain a wavelength region averaged value for $\mathrm{A}_{e}$ by knowing the relative wavelength response of the instrument and determining the absolute response to some known source.

\section{Quantitative Calibration}

\subsection{General Description}

The concept of FUV imaging is illustrated in Figure 1. On the left, we schematically illustrate the atmosphere with its main constituents $\mathrm{O}_{2}, \mathrm{~N}_{2}$ and $\mathrm{O}$. The spectra of the emissions are influenced by the mixing ratio of the excited species, the lifetime of the excited state related to the average collision frequency and the opacity of the atmosphere above the interaction region. The resultant spectra of the auroral emissions received by a satellite instrument are critically dependent on the mixing ratio of $\mathrm{O}_{2}, \mathrm{~N}_{2}$ and $\mathrm{O}$ near the height of the auroral photon emission which is in turn dependent on the energy of the primary particle. This is the frequently used methodology for the spectroscopic determination of auroral primary energies (Germany et al., 1994).

The rectangles in the middle illustrate the various stages of processing with the results or measurable illustrated to the right of each rectangular box. On the rightmost column we define the various calibration quantities as $X_{1}, X_{2}, X_{3}$, and $\mathrm{X}_{4}$.

The top box represents the auroral particles, which are characterized by differential energy spectra and pitch angle distributions. Customarily, the energy spectra are given in terms of particle fluxes in various energy intervals that can be in- 


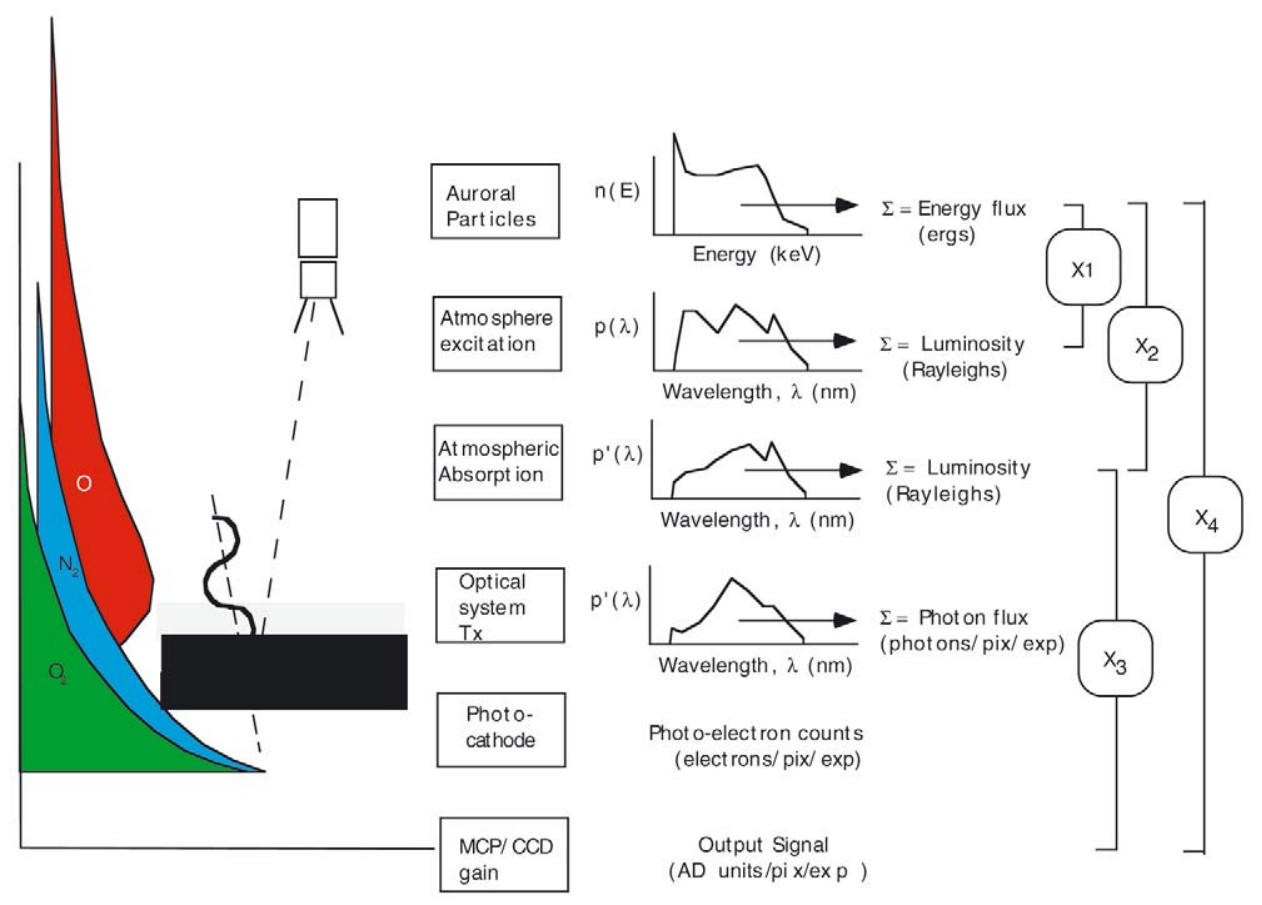

Figure 1. Schematic representation of the components of the instrument calibration chain (rectangles) from auroral particle inputs (top) through atmospheric interactions to the output of the instrument (bottom). The transfer functions between the various stages are represented by X-s.

tegrated into a total deposited energy flux $\Sigma$ in $\mathrm{mW} / \mathrm{m}^{2}$. The second box represents the auroral excitation process by primary and secondary particles. For some wavelength emissions the lower part of the atmosphere is fairly opaque and the third box represents the filtering by atmospheric absorption.

The following 3 boxes represent the performance of the instrument starting with the transmission properties of the optics. The current produced by the photocathode is a result of the convolution of the emitted spectral profile with the optical and photo emissive responses of the instrument. That electron signal is usually amplified by a micro channel plate or in the solid-state amplifier of a CCD. The action of the instrument can thus be modeled from the input of the optical system to the output of the final signal amplifier and is described as $\mathrm{X}_{3}$.

Our instrument calibration activities are aimed at quantifying the properties of $\mathrm{X}_{3}$. The modeling attempts to relate the output signal of the instrument to the particle precipitation fluxes and energies are finally shown by $\mathrm{X}_{4}$.

Using the above concepts we proceed in the following way. The transfer function $\mathrm{X}_{3}$ can be modeled or experimentally characterized by making in-flight calibration measurements of stars with well-known UV emission spectra. By performing model calculations of different particle energies and fluxes and obtaining the atmospheric emission response the transfer functions without $\left(\mathrm{X}_{1}\right)$ or with $\left(\mathrm{X}_{2}\right)$ at- 
mospheric absorption were characterized. By applying the modeled and calibrated properties of the instrument we can obtain the characteristic of the transfer function $\mathrm{X}_{4} . \mathrm{X}_{4}$ can also be obtained empirically from direct comparisons of particle detector satellite over-flight data with simultaneous imaging data.

By making observations of several B stars with the FUV instruments we determined the response to known FUV fluxes as measured by the International Ultraviolet Explorer (IUE) observatory (Cassatella et al., 2000). These stellar calibrations were obtained on 19th June, 2000 (Gladstone et al., 2000). We consider these results as the baseline sensitivity for the instruments with a normalization factor of unity. Future gain variations can then be expressed by a factor relative to this number.

\subsection{Si-12 CALIBRATiOnS}

The FUV spectra of the primarily B stars are broadband continua which peak at about $110 \mathrm{~nm}$ and are provided in photons $/ \mathrm{cm}^{2} / \mathrm{s} / \mathrm{nm}$ by the IUE archive. Here we use only high-dispersion, large aperture, short wavelength IUE spectra. Measurements with a point source can be easily interpreted to yield extended-source sensitivity, as long as the field of view of the instrument is well known from laboratory calibrations. It is then possible to convolve the normalized spectral transmission profiles obtained in pre-flight laboratory calibrations with the IUE spectrum. The result is integrated to find the number of Rayleigh for a thin line spectrally located at the peak of the SI-12 transmission that would produce the same output signal.

Figure 2 shows the SI-12 response to various stars. It is interesting to compare this result with that of our prediction from laboratory absolute calibrations. (Mende et al., 2000) Table III shows that the expected counts per resolution cell for the $\mathrm{T}_{\text {exp }}=5 \mathrm{sec}$ observation of a $1 \mathrm{kR}$ source was 18 counts $/ \mathrm{kR}$. This compares quite well to the in-flight stellar calibration result of 23.5 counts/kR shown in Figure 2.

A $1 \mathrm{kR}$ aurora produces $10^{9} / 4 \pi$ photons $/ \mathrm{sr} / \mathrm{cm}^{2} / \mathrm{s}$. In a pixel of solid angle $4.9 \times$ $10^{-6} \mathrm{sr}, 392$ photons per second or 1960 photons in the 5-second exposure are collected. The equivalent aperture therefore is $23.5 / 1960=0.012 \mathrm{~cm}^{2}$. This should be compared with the last but one column of Table III of (Mende et al., 2000) which is $0.01 \mathrm{~cm}^{2}$. This permits us to estimate the overall optical transmission and photo-cathode counting efficiency of the SI-12 from its known aperture $\left(\approx 1 \mathrm{~cm}^{2}\right)$ and it was found to be $1.2 \%$.

Early in the SI-12 design it was realized that the periodic slit grille allows for some transmission around $120 \mathrm{~nm}$, where there is a strong triplet of atomic nitrogen emission (Mende et al., 2000). The design was optimized for maximum performance around $121.6 \mathrm{~nm}$ and at least less than $10 \%$ transmission around $120.0 \mathrm{~nm}$. The first two years of operation show that the SI-12 is very insensitive to the $120.0 \mathrm{~nm}$ emission, which does not produce considerable contribution to the images except near the subsolar limb (Immel et al., 2000). 


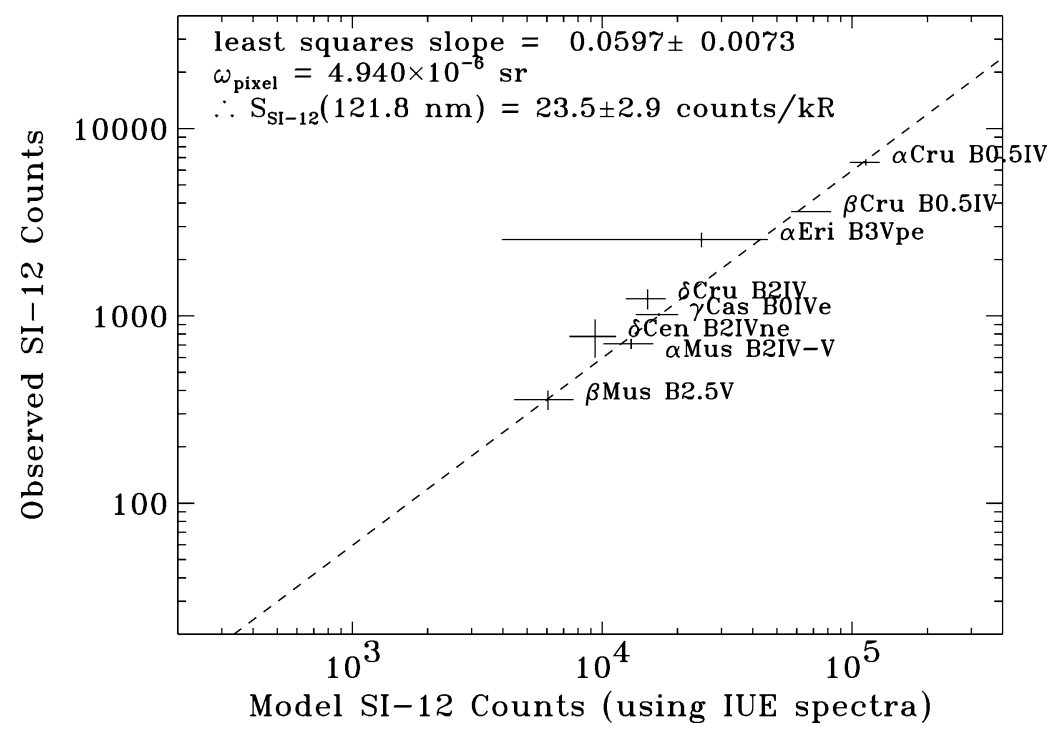

Figure 2. Stellar calibration of the SI-12 channel. The average and standard deviation of the SI-12 counts for each star were determined from three separate measurements, and the IUE flux error was estimated as the average uncertainty over the SI-12 bandpass. The HV setting was $4200 \mathrm{~V}$. The temperatures for the collimator and grid were $1^{\circ} \mathrm{C}$.

\subsection{WIC CALIBRATIONS}

Figure 3 shows a laboratory spectrum of the FUV emission from $\mathrm{N}_{2}$ after electron bombardment (Ajello and Shemansky, 1985). It also shows the relative spectral response of WIC with a maximum at $150 \mathrm{~nm}$ and low sensitivity below $140 \mathrm{~nm}$ and above $180 \mathrm{~nm}$.

The measurements using astronomical data are shown in Figure 4 and accordingly the WIC produces $612.6 \mathrm{AD}$ units per $\mathrm{kR}$ during a 10 second exposure at its peak sensitivity of $150 \mathrm{~nm}$ emission (Gladstone et al., 2000). From simple geometric considerations WIC has a pixel solid angle $1.375 \times 10^{-6} \mathrm{sr}$ and the photon arrival rate is 110 photons $/ \mathrm{s} / \mathrm{cm}^{2} / \mathrm{pixel} / \mathrm{kR}$ or during the nominal exposure time of the WIC (10 second) is 1100 photons $/ \mathrm{cm}^{2} / \mathrm{pixel} / \mathrm{kR}$. Thus the measured photons to ADA count conversion is $613 / 1100=0.56$ at the peak of its response $(150 \mathrm{~nm})$. For comparison the WIC was calibrated in terms of photons per $\mathrm{cm}^{2}$ at the input and a peak response of 0.63 were determined.

\subsection{SI-13 CALIBRATIONS}

The results of the SI-13 stellar calibration are given in Figure 5 and it shows an overall sensitivity of 15.3 counts $/ \mathrm{kR}$. It is interesting to compare his result with that of our predictions from ground based calibrations. (Mende et al., 2000) Table III shows that the counts per resolution cell for a $1 \mathrm{kR}$ source are 13 counts/kR. This compares with the in flight calibration number of 15.3 counts/kR above. 


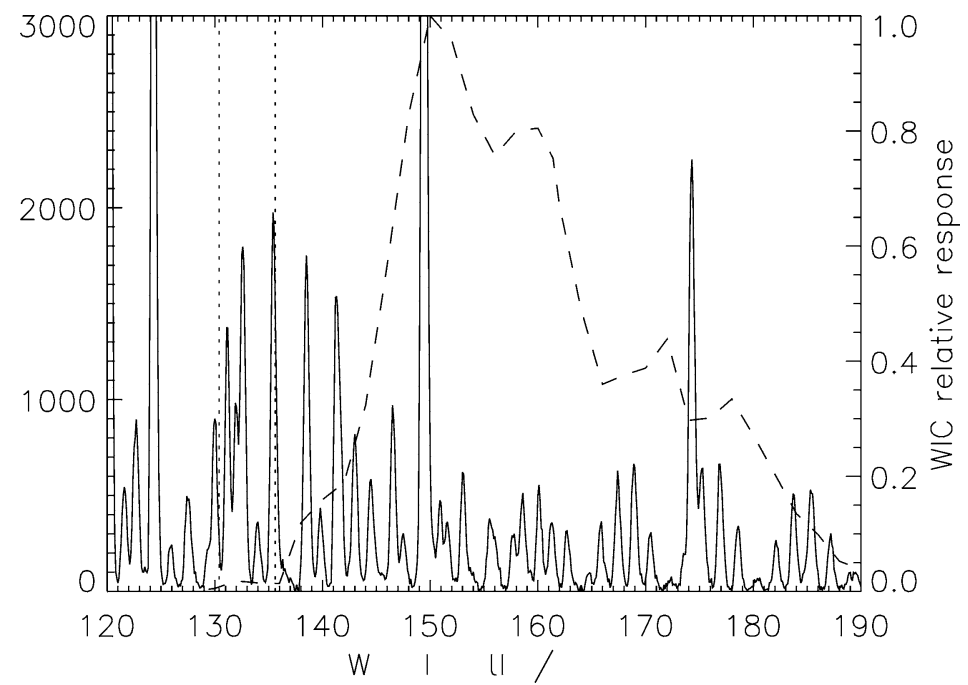

Figure 3. Laboratory spectrum of FUV emission from $\mathrm{N}_{2}$ (Ajello and Shemansky, 1985) and WIC relative spectral response (dashed line). Dotted lines indicate the location of oxygen emissions at $130.4 \mathrm{~nm}$ and $135.6 \mathrm{~nm}$.

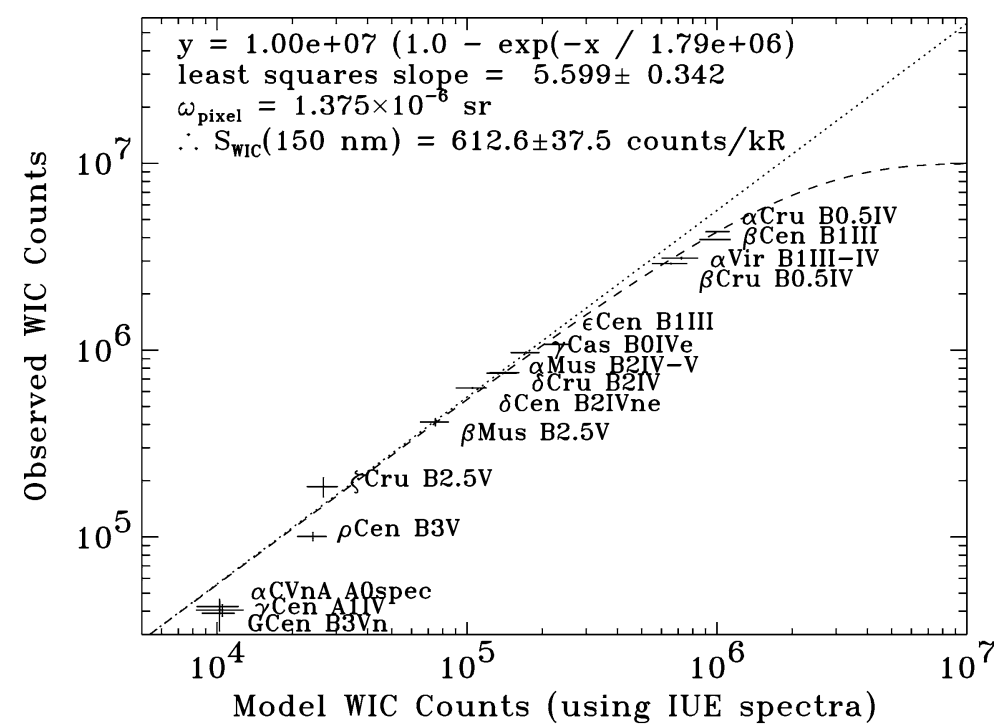

Figure 4. Stellar calibration results for the WIC instrument. At very large count rates the WIC response is non-linear, so an asymptotic function was least-squares fit to the results, yielding a sensitivity (in the linear response region) of $612 \mathrm{AD}$ units $/ \mathrm{kR}$ at the peak response wavelength of $150 \mathrm{~nm}$ (see Figure 3). HV settings were $1100 \mathrm{~V}$ for the MCP and $4000 \mathrm{~V}$ for the phosphor. The temperatures were $-6^{\circ} \mathrm{C}$ for the secondary mirror and $1{ }^{\circ} \mathrm{C}$ for the detector. 


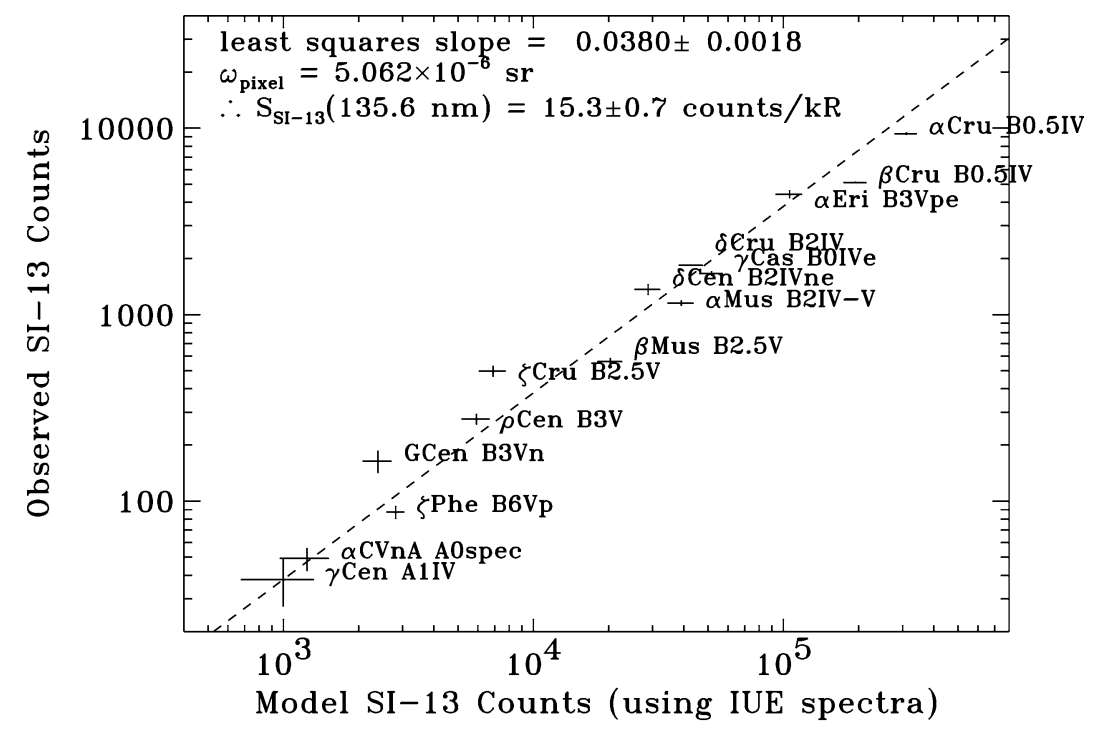

Figure 5. Stellar calibration results for the SI-13 instrument. A least-squares fit to the results yields a sensitivity of 15.3 counts/kR at the peak response wavelength of $135.6 \mathrm{~nm}$. The HV setting was $4100 \mathrm{~V}$. The collimator and grid temperatures were $1^{\circ} \mathrm{C}$.

In order to calculate the equivalent aperture of the instruments we need to calculate the expected photon efficiency. A $1 \mathrm{kR}$ aurora produces $10^{9} / 4 \pi$ photons/ $\mathrm{sr} / \mathrm{s} / \mathrm{cm}^{2}$ and in a pixel of solid angle $5.062 \times 10^{-6} \mathrm{sr}$ it produces 405 photons $/ \mathrm{s} / \mathrm{cm}^{2}$ or 2024 photons $/ \mathrm{cm}^{2}$ in the 5 -second exposure. The equivalent aperture therefore is $15.3 / 2024=0.0076 \mathrm{~cm}^{2}$. This should be compared with the last but one column of Table III that is 0.008 . Since the combined aperture of the SI slits is about $1 \mathrm{~cm}^{2}$ it shows that the overall counting efficiency of the SI-13 is $0.8 \%$.

\section{4. $X_{1}$ and $X_{2}$ Emission Modeling}

To calculate the overall transfer function $\mathrm{X}_{4}$ of the instruments it is necessary to assume a particle spectrum and model the aurora production and the instrument response (Gérard et al., 2000; Hubert et al., 2001). First, as an intermediate step in this process, it is necessary to calculate the atmospheric response to precipitating particle fluxes. In its full detail this response is a wavelength dependent function. However it is possible to integrate the function and express the results as emissions in Rayleigh. In Figure 1 we denoted the partial transfers as $X_{1}$ and $X_{2}$.

The methodology to calculate the excitation rates rests on the combination of two transport models. Those respectively describe the interaction of electron and proton beams with the atmosphere. Together they account for collisional energy degradation, the generation of a neutral $\mathrm{H}$ beam following collisions and the pro- 
TABLE I

Atmospheric response to proton precipitation. The table is in emitted Rayleigh of Lyman- $\alpha$, LBH (combination of LBH and NI, see text), 1356, and 1304 given before and after atmospheric absorption for $1 \mathrm{~mW} / \mathrm{m}^{2}$ proton precipitation.

\begin{tabular}{lrrrrrrl}
\hline$<E>(\mathrm{keV})$ & Ly- $\alpha$ & LBH & LBH abs & 1356 & 1356 abs & 1304 & 1304 abs \\
\hline 0.47 & 12770 & 762 & 751 & 96 & 95 & 123 & 123 \\
2.00 & 9000 & 1690 & 1660 & 191 & 188 & 334 & 333 \\
8.00 & 4770 & 3070 & 2930 & 298 & 289 & 626 & 625 \\
25.0 & 2360 & 3460 & 3240 & 292 & 277 & 690 & 688 \\
46.7 & 1630 & 3326 & 3068 & 259 & 243 & 643 & 641 \\
\hline
\end{tabular}

duction of secondary electrons (Solomon et al., 1988; Solomon, 2000; Gérard et al., 2000).

Details of the kinetic code for the simulation of the proton Lyman- $\alpha$ line profile and the interaction of energetic protons with atmospheric particles are given in (Gérard et al., 2000; Hubert et al., 2001). This code also takes into account the stochastic nature of collision scattering to properly describe the behavior of high-energy protons, which collide with the atmospheric particles and change their direction following a probabilistic distribution of the scattering angle. The model is self-consistent, as all sources for the beam spreading (collisional, geometrical, magnetic mirroring) are included.

The resulting volume excitation rates for the $\mathrm{N}_{2} \mathrm{LBH}$ bands and the nitrogen line emission are calculated including all collisional excitation processes. The emerging intensities are obtained by integration along the line of sight. The result of this simulation was then convoluted with the instrument passbands to calculate the expected signal in the three FUV images. The simulations indicate that the LBH and $135.6 \mathrm{~nm}$ emissions may contain significant contributions from proton excitation (Lummerzheim et al., 2001). High-energy protons may penetrate to lower altitudes where the $\mathrm{O}_{2}$ Schumann-Runge absorption continuum reduces the observed LBH intensity. The full simulation code includes this absorption assuming moderately disturbed conditions during solar maximum, but quantitative analysis of auroral energy using global observations will always suffer from the unknown peak energy of precipitating protons unless higher spectral resolution images are available.

Doppler shifted Lyman- $\alpha$ aurora is generally subject to less atmospheric absorption. Most proton interactions take place at high altitude where the density of absorbers is minimal. Also, $\mathrm{O}_{2}$ is not a very good absorber at $121.6 \mathrm{~nm}$ (Meier, 1991). Therefore, for Doppler shifted Lyman alpha $X_{1}$ and $X_{2}$ are the same. The results of the proton model calculations are in Table I. The Lyman- $\alpha$ values are the same as given in Table I of (Gérard et al., 2001). 

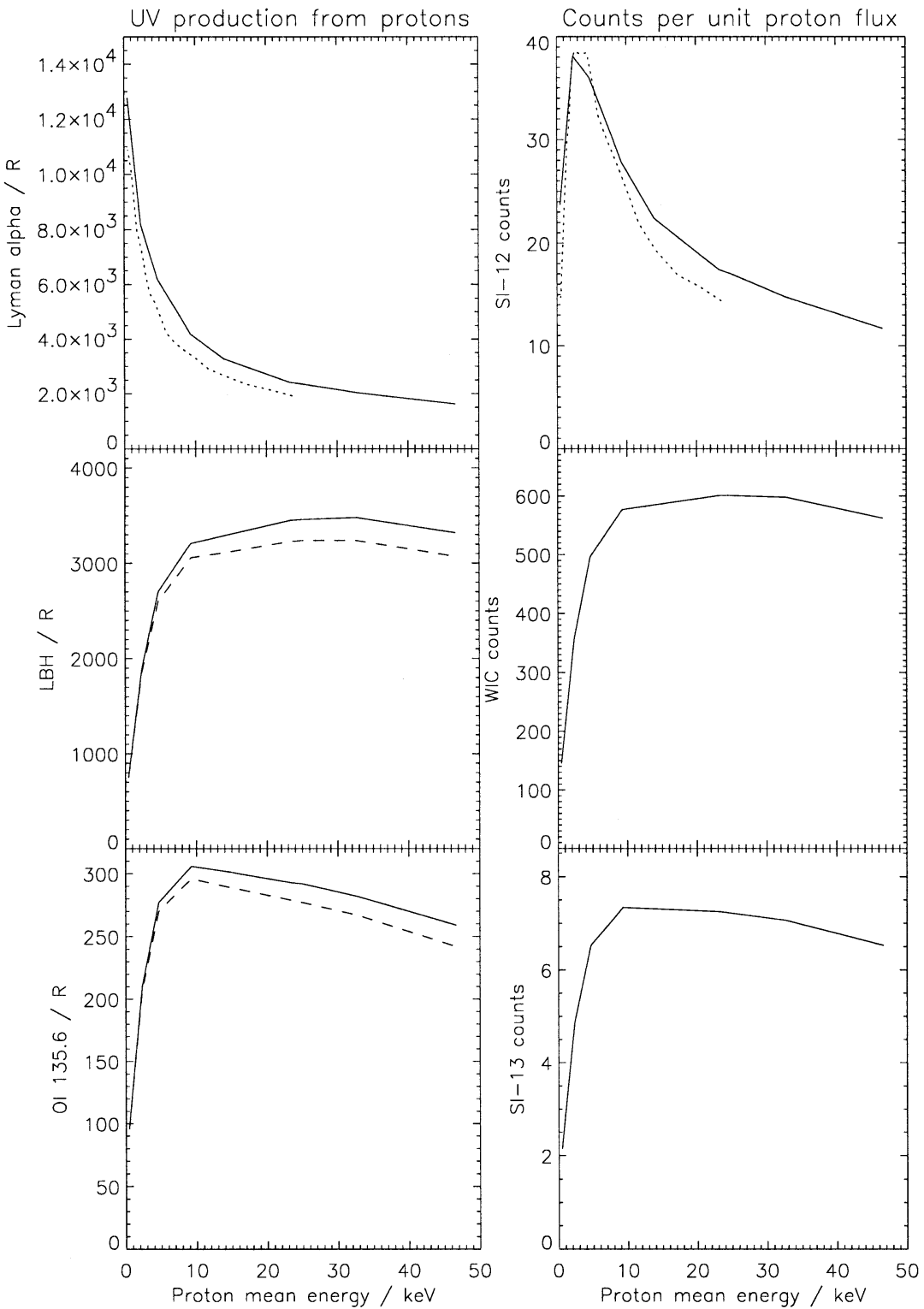

Figure 6. Predicted emission intensities from proton precipitation (left, from Table I, dashed line with absorption) and the corresponding count rates for $1 \mathrm{~mW} / \mathrm{m}^{2}$ energy flux (right, data of Tables III, V, VIII). Dotted line for monoenergetic beams, solid line for kappa-distribution. 


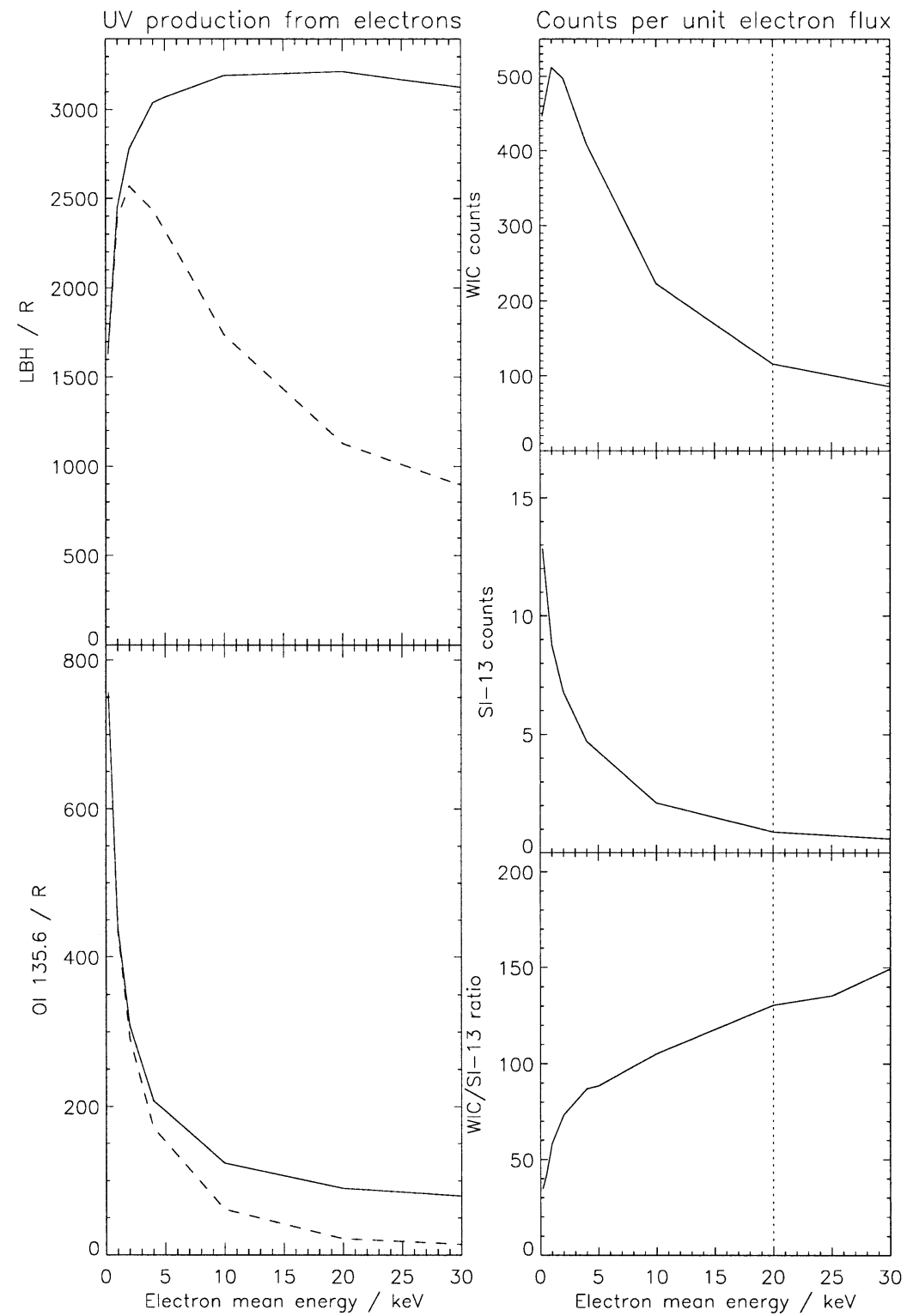

Figure 7. Predicted emission intensities from electron precipitation (left, from Table II, dashed line with absorption) and the corresponding count rates for $1 \mathrm{~mW} / \mathrm{m}^{2}$ energy flux (right, data of Tables IV, VII) and the ratio of both for unit electron energy fluxes. 
TABLE II

Atmospheric response to electron precipitation. The table is in emitted Rayleigh with $\mathrm{LBH}, 135.6$ and 130.4 given with and without atmospheric absorption for $1 \mathrm{~mW} / \mathrm{m}^{2}$ electron precipitation.

\begin{tabular}{lrlrlrl}
\hline$<E>(\mathrm{keV})$ & LBH & LBH abs. & 1356 & 1356 abs. & 1304 & 1304 abs. \\
\hline 0.20 & 1630 & 1629 & 757 & 756 & 2908 & 2907 \\
0.50 & 1940 & 1910 & 638 & 635 & 2420 & 2420 \\
1.00 & 2450 & 2390 & 440 & 434 & 1607 & 1606 \\
5.00 & 3070 & 2320 & 194 & 153 & 630 & 621 \\
10.0 & 3194 & 1738 & 124 & 61 & 321 & 305 \\
25.0 & 3170 & 1010 & 85 & 18 & 132 & 116 \\
\hline
\end{tabular}

Details for the electron aurora simulations are given in (Hubert et al., 2001; Hubert et al., 2002). The calculated volume emission rates were then integrated for a nadir observation from a spacecraft and the total emission intensities are given in Table II. Please note that the values shown in figure 5 of the paper (Hubert et al., 2001) reflect pure LBH emission from $\mathrm{N}_{2}$ molecules. Everywhere in this paper we will consider LBH as the combination of LBH from $\mathrm{N}_{2}$ and atomic nitrogen lines. As the instrument can not distinguish photons from different sources we need to include all emissions in the respective wavelength range, even the small contribution from OI. Within the whole FUV region atomic nitrogen lines increase the total number of LBH photons by a factor of 2.6, and especially the NI line at $149.3 \mathrm{~nm}$ is important for our WIC observations because it is close to the sensitivity maximum (Figure 3).

The emission simulation also requires a model for the altitude distribution of the atmospheric constituents $\mathrm{O}_{2}, \mathrm{~N}_{2}$, and O. All calculations used the MSIS-90 model for moderately disturbed geomagnetic conditions (Hedin, 1991). The input particle distributions were isotropic Maxwellian distributions for electrons and kappa-functions with $\kappa=3.5$ for protons. Kappa-functions with their high-energy tail are better suited to describe measured proton distributions than the more symmetric Maxwellian functions (Gérard et al., 2000). A unit starting energy flux of $1 \mathrm{~mW} / \mathrm{m}^{2}$ at $600 \mathrm{~km}$ altitude within the loss cone was used. If these results were to be compared with flux values at other altitudes (for instance satellite measurements at $4000 \mathrm{~km}$ ), a mapping factor of the converging magnetic field needs to be applied.

Figures 6 and 7 summarize the results from Tables I and II. The left column shows the excitation efficiencies for the different FUV emissions from electron and proton impact with different mean energies and equal energy flux of $1 \mathrm{~mW} / \mathrm{m}^{2}$. The top two panels also show results with a monoenergetic proton distribution (Gérard et al., 2001). As monoenergetic distributions are lacking the high energy tail their 


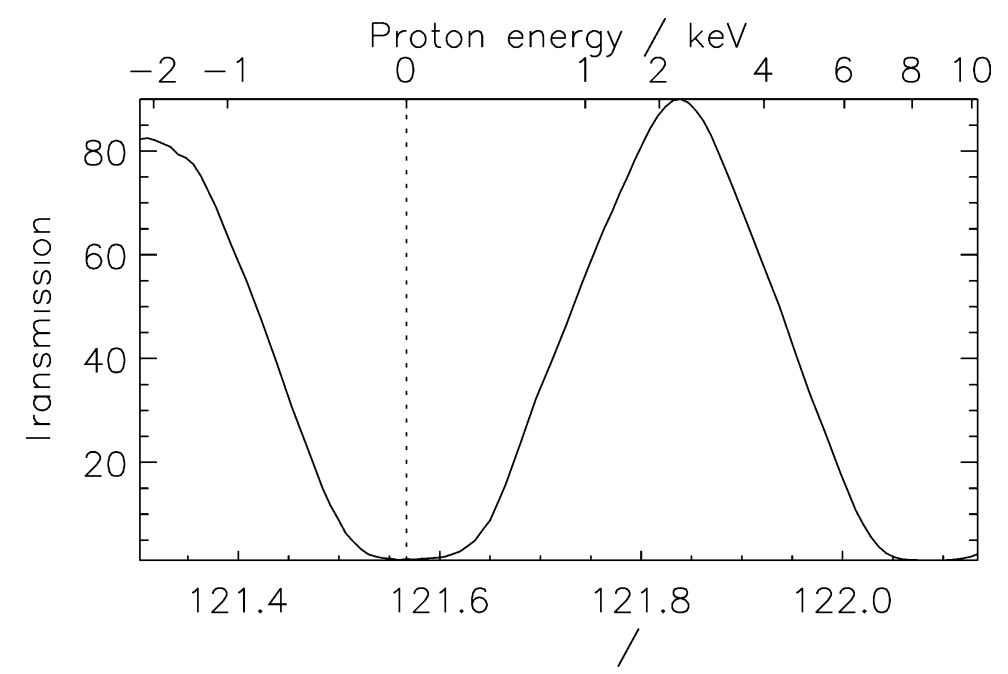

Figure 8. Transmission of the SI-12 instrument around the rest wavelength of hydrogen Lyman- $\alpha$ (121.567 nm, dotted vertical line). The top axis marks proton energies in $\mathrm{keV}$ for the corresponding Doppler shift assuming a proton moving straight away from the observer.

overall Doppler shift is smaller than the kappa-distributions and we get fewer SI-12 counts for the same mean energy and energy flux.

\section{Modeling of the Full $\mathbf{X}_{4}$ Transfer Function}

The SI-12 has a periodic pass band due to the slit grille in the instrument with minimal transmission at the rest wavelength of hydrogen Lyman- $\alpha$ (Mende et al., 2000). Figure 8 shows the high resolution ray tracing together with a scale of proton energies which would correspond to these Doppler shifted wavelengths assuming a straight motion of energetic protons away from the observer. All model calculations were performed with proton energy distributions with certain mean energies thus having protons with smaller and higher individual energies. Furthermore, photons from particles with some original pitch angle will always have smaller Doppler shift then those from particles moving straight away. The final response of the instrument to proton precipitation is therefore the combination of original differential energy distribution, pitch angle distribution, deceleration of the protons due to atmospheric interaction, change of charge-exchange cross section with energy, and altitude-dependent density distribution of the atmosphere. It turns out, that for unit precipitating energy flux, SI-12 is most sensitive to an isotropic distribution of protons with 3-4 keV mean energy.

The comparison between Tables II and IV allows us to calculate the efficiency of WIC in counts per actual kR as a function of the mean energy (Table VI). For the unabsorbed emission we get the expected result, that the count rate is independent 


\section{TABLE III}

SI-12 response for protons of various mean energies. The column P2 refers to Figure 10. Counts are given for nominal exposure time of $5 \mathrm{~s}$.

\begin{tabular}{clcll}
\hline$<E>(\mathrm{keV})$ & Counts per $1 \mathrm{~mW} / \mathrm{m}^{2}, P 2$ & Rayleigh & Counts $/ \mathrm{kR}$ & $\mathrm{A}_{e}\left(\mathrm{~cm}^{2}\right)$ \\
\hline 0.47 & 23.7 & 12770 & 1.86 & 0.0009 \\
2.00 & 35.6 & 9000 & 3.95 & 0.0020 \\
8.00 & 30.2 & 4770 & 6.33 & 0.0032 \\
25.0 & 17.0 & 2360 & 7.20 & 0.0037 \\
46.7 & 11.7 & 1630 & 7.18 & 0.0037 \\
\hline
\end{tabular}

TABLE IV

WIC response in counts to $1 \mathrm{~mW} / \mathrm{m}^{2}$ electron energy flux. The column E1 refers to Figure 10.

\begin{tabular}{cccccccc}
\hline $\begin{array}{c}<E> \\
(\mathrm{keV})\end{array}$ & LBH & LBH abs. & 1356 & 1356 abs. & 1304 & 1304 abs. & $\begin{array}{l}\text { Total abs. } \\
\text { E1 }\end{array}$ \\
\hline 0.20 & 295 & 295 & 45.4 & 45.4 & 106 & 106 & 446 \\
0.50 & 351 & 344 & 37.6 & 37.5 & 88.6 & 88.6 & 470 \\
1.00 & 443 & 427 & 25.4 & 25.1 & 58.8 & 58.8 & 511 \\
5.00 & 556 & 346 & 9.65 & 8.15 & 23.1 & 22.7 & 377 \\
10.0 & 579 & 208 & 7.43 & 3.68 & 11.8 & 11.1 & 223 \\
25.0 & 575 & 95 & 1.93 & 0.89 & 4.84 & 4.24 & 101 \\
\hline
\end{tabular}

TABLE V

WIC counts in response to $1 \mathrm{~mW} / \mathrm{m}^{2}$ proton energy flux. The column P1 refers to Figure 10.

\begin{tabular}{cclcccl}
\hline$<E>(\mathrm{keV})$ & LBH & LBH abs & 1356 & 1356 abs & 1304 abs & Total abs, P1 \\
\hline 0.47 & 138 & 135 & 5.78 & 5.73 & 4.51 & 145 \\
2.00 & 307 & 296 & 11.4 & 11.3 & 12.2 & 319 \\
8.00 & 556 & 514 & 17.9 & 17.3 & 22.9 & 554 \\
25.0 & 627 & 559 & 17.5 & 16.6 & 25.2 & 601 \\
46.7 & 603 & 524 & 15.5 & 14.6 & 23.5 & 562 \\
\hline
\end{tabular}


TABLE VI

WIC response in ADA units per $\mathrm{kR}$ for electrons.

\begin{tabular}{lll}
\hline$<E>(\mathrm{keV})$ & LBH & LBH abs. \\
\hline 0.2 & 181 & 181 \\
0.5 & 181 & 180 \\
1.0 & 181 & 179 \\
5.0 & 181 & 149 \\
10.0 & 181 & 119 \\
25.0 & 181 & 94 \\
\hline
\end{tabular}

\section{TABLE VII}

SI-13 counts as a function of the mean energy for a $1 \mathrm{~mW} / \mathrm{m} 2$ energy flux of electron precipitation. The column E3 refers to Figure 10.

\begin{tabular}{cclcccc}
\hline $\begin{array}{c}<E> \\
(\mathrm{keV})\end{array}$ & LBH & LBH abs. & 1356 & 1356 abs. & $\begin{array}{l}\text { Total abs. } \\
\text { E3 }\end{array}$ & $\begin{array}{l}\text { per kR } \\
1356\end{array}$ \\
\hline 0.20 & 1.59 & 1.59 & 11.2 & 11.2 & 12.8 & 14.8 \\
0.50 & 1.89 & 1.86 & 9.46 & 9.42 & 11.3 & 14.8 \\
1.00 & 2.38 & 2.32 & 6.53 & 6.43 & 8.75 & 14.8 \\
5.00 & 2.99 & 2.00 & 2.87 & 2.26 & 4.26 & 14.8 \\
10.0 & 3.11 & 1.20 & 1.83 & 0.91 & 2.11 & 14.8 \\
25.0 & 3.09 & 0.47 & 1.26 & 0.27 & 0.74 & 14.8 \\
\hline
\end{tabular}

of the mean energy of the incoming electrons. For higher energies the number of counts is reduced due to atmospheric absorption. Energetic protons act similarly to low energy electrons (Lummerzheim et al., 2001). Therefore, the response of WIC in terms of counts per $\mathrm{kR}$ of LBH from protons is for all energies almost identical to the response to $0.2-1.0 \mathrm{keV}$ electrons.

\section{Temporal Variations in Instrument Sensitivity}

The stellar calibrations were performed on the 19th of June 2000. Laboratory tests and experience during the first months of in-orbit operations showed some sensitivity of the SI to temperature changes. These gain variations are monitored and are expressed as a correction factor relative to this day. 
TABLE VIII

SI-13 Count rates for protons for $1 \mathrm{~mW} / \mathrm{m}^{2}$ energy flux. The column P3 refers to Figure 10.

\begin{tabular}{ccllll}
\hline$<E>(\mathrm{keV})$ & LBH & LBH abs. & 1356 & 1356 abs. & Total abs., P3 \\
\hline 0.47 & 0.74 & 0.73 & 1.43 & 1.41 & 2.14 \\
2.00 & 1.65 & 1.61 & 2.83 & 2.78 & 4.39 \\
8.00 & 2.99 & 2.81 & 4.42 & 4.28 & 7.09 \\
25.0 & 3.37 & 3.09 & 4.32 & 4.11 & 7.20 \\
46.7 & 3.24 & 2.91 & 3.84 & 3.60 & 6.51 \\
\hline
\end{tabular}

Two ways of in-orbit gain monitoring were used, full earth observations and star observations. At apogee the earth fills the full field of view of all three FUV imagers. The total of all counts in the images from apogee should only show seasonal variations and slow changes due to the apogee precession $\left(45^{\circ}\right.$ per year). A full model of the dayglow intensity and observation geometry could then be used to relate temporal deviations of the expected signal from measurements with gain changes (Gladstone, 1994). However, this method proved to be not accurate enough because large geomagnetic storms (for instance Bastille Day 2001) caused dramatic increases in auroral intensity, which also increased the total observed signal without gain changes.

Three different stars were chosen which regularly move through the imager field of view and their total signal was recorded over time. 30 dedicated minutes of star pointing time are scheduled once per week to increase the total observation time of the test stars. The total signal of these stars is then related to the signal during the all-sky survey on June 19, 2000 and every deviation from a ratio of 1.0 is applied as a correction factor to the intensity in the whole image. Figure 9 shows the result for observations of Delta Crux (a bright ultraviolet B-star). The ratio of total star counts with regard to 2000-171 and the temperature of the WIC-detector and the SI-13 pre-amplifier are shown. The changing angle between the spacecraft main axis and the direction to the sun causes high temperatures around May 15 and August 23, and lows around January 5. The WIC instrument is not sensitive to temperature changes, however we see a slow and steady sensitivity decrease over the first 1.75 years of the mission. The $74 \%$ gain increase after high voltage change (Figure 9, day 353) corresponds well to the pre-flight calibration of $77 \%$.

Obvious changes in the SI-gain are caused by high temperatures, which most likely influence the performance of the electronics. Heater operations have to be adjusted during the warm-periods, and high voltage changes can partly counteract these decreases. 

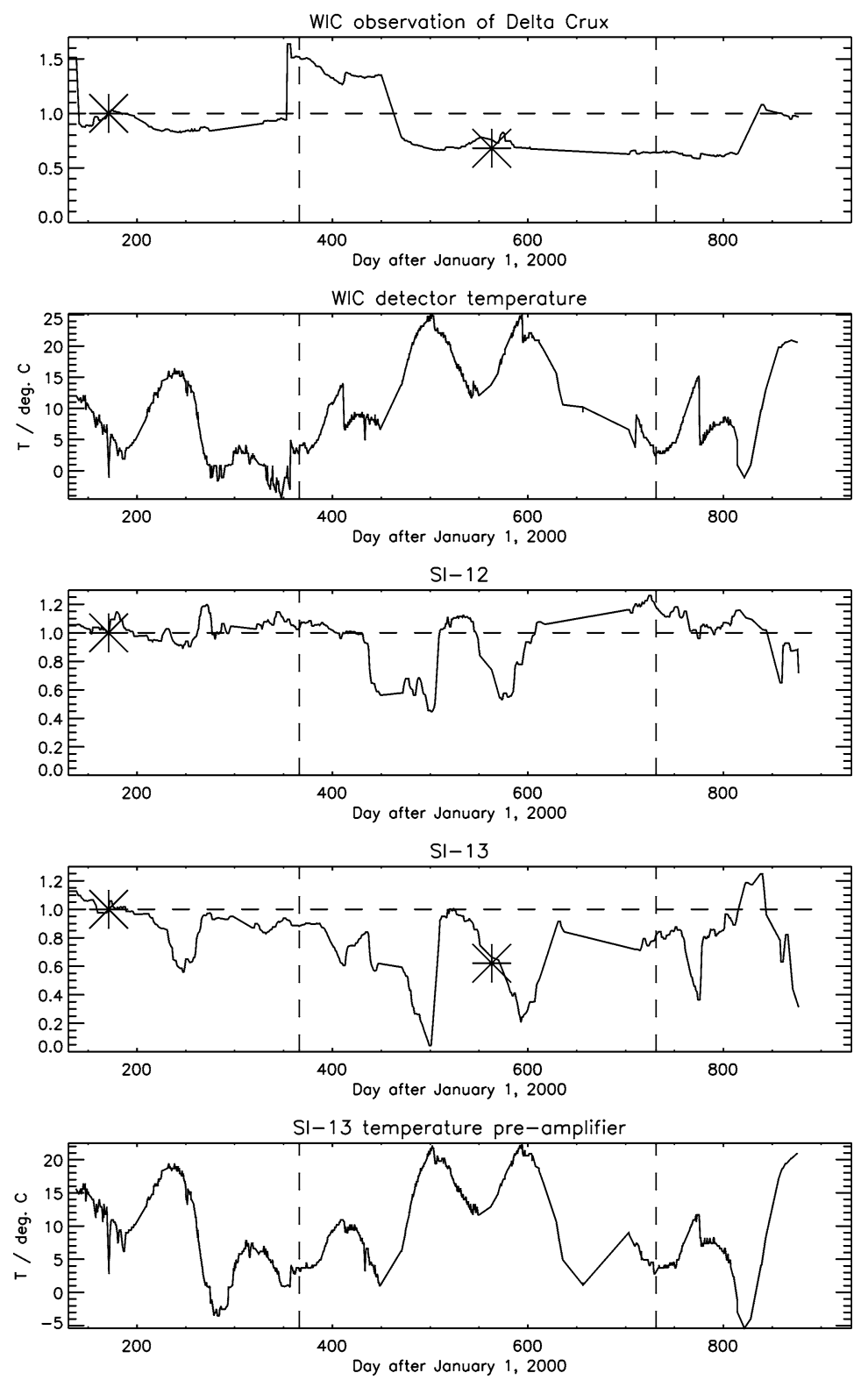

Figure 9. Stellar observations of Delta Crux with IMAGE-FUV and corresponding temperatures of most relevant instrument parts. The top panel shows the ratio of total star counts in WIC related to the calibration on June 19, 2000. During the very first days, on days 352-450, and after day 830 a higher high voltage was used. The second panel shows the temperature of the WIC detector. Panels three and four show the ratios for SI-12 and SI-13, respectively. The last panel shows the temperature of the SI-13 pre-amplifier. 


\section{Image Pre-processing}

In order to extract quantitative results of the aurora from images these images have to be flat-fielded and the dayglow has to be removed. Dayglow subtraction is achieved using a model based on the response of the individual instruments to quiet-time dayglow observations. In this model, the dayglow brightness and subsequent instrument response depend on the solar and spacecraft zenith angles at the point intersecting the line of sight observation at an assumed emission altitude. This is similar to the model created for Dynamics Explorer 1 FUV images (Immel et al., 2000). Adjustments for instrument sensitivity and solar 10.7-cm flux are applied.

Flatfield corrections are determined using low altitude images, where the field of view of the imagers covers sunlit middle latitude locations. The instrumental response to the FUV dayglow signature is normalized to the mean solar zenith angle (using the aforementioned dayglow model), and then normalized to the instrument response at the image center. This reduces the image to values around unity. Deviations from unity are fitted with a parabolic function (in the case of WIC), or simply kept in a lookup table (in the case of SI), for later use in normalizing images obtained from any other vantage point to equal response/pixel.

\section{Summary of Reduction to Particle Energies and Fluxes}

The columns P1, P2, and P3 in Tables III, V, VIII provide the contribution of instrument signal from proton excitation for WIC, SI-12, and SI-13, respectively. Similarly the columns E1 and E3 in Tables IV and VII provide the expected signal in WIC and SI-13 from pure electron excitation. Using these numbers, Figure 10 shows the flow chart, how quantitative estimates can be obtained.

Inputs are the three images from WIC, SI-12 and SI-13 after they have been properly pre-processed and corrected for temporal changes. As we have at least 4 unknowns (energy and flux of protons and electrons), we need to estimate at least one of them. Generally, we estimate the proton mean energy by using the corresponding model predictions (Hardy et al., 1989; Hardy et al., 1991). We can then determine the proton energy flux from the SI-12 images. Then, the SI-13 and WIC images are corrected for the proton contribution leaving the signal from pure electron precipitation. These values can be used to estimate the mean electron energy by applying the ratios from Figure 7 . The mean electron energy is then the input to the final step of energy flux estimates. This energy flux can be estimated from both SI-13 and WIC, which allows for some cross check. Generally the WICresults should be used as they show higher count rates with reduced statistical uncertainties and these images also represent the better spatial resolution.

The results of such estimates are still somewhat uncertain as many simplifications are used. Slightly better results can be obtained with pure proton (afternoon 


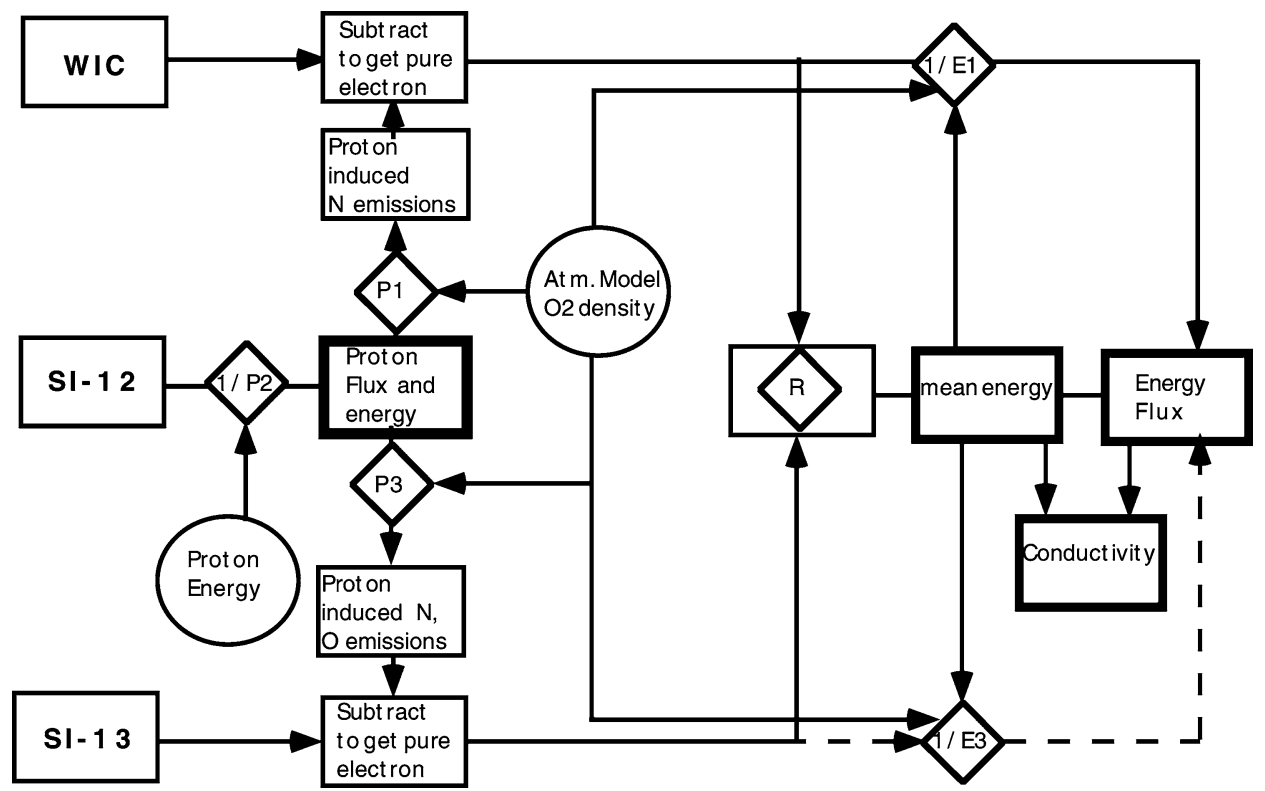

Figure 10. How to use the IMAGE FUV counts to obtain particle mean energies and energy fluxes.

sector) and pure electron cases (when SI-12 does not show any signal). After all an accuracy of about $50 \%$ can be expected as will be demonstrated in the following chapter.

\section{Validation of the Model with Satellite Overpasses}

The scheme of quantitative analysis was first verified with satellite data during simultaneous measurements by FAST and observations by FUV. On June 24, 2000 FAST crossed the auroral oval (Figure 11) and Figure 12 shows the measurements in highest time resolution of $0.6 \mathrm{~s}$. Depending on the altitude of the satellite the velocity changes, but in general FAST traveled about $2 \mathrm{~km}$ between two measurement points. This is much smaller then the spatial resolution of the FUV-observations (50 or $100 \mathrm{~km}$ ) and therefore the satellite measurements had to be averaged over some time.

The FAST particle measurements in the loss cone were used as input to a full-code simulation of the production of auroral FUV emission and the expected signal in the FUV instrument (Frey et al., 2001; Gérard et al., 2001). The results confirmed the general good agreement between simulation results and FUVobservations. For the WIC observations (Figure 13) it was demonstrated that under certain circumstances high proton fluxes may produce significant amounts of auroral FUV emission (Frey et al., 2001). Further investigation confirmed the general good agreement for SI-12 (Figure 14) (Gérard et al., 2001). 


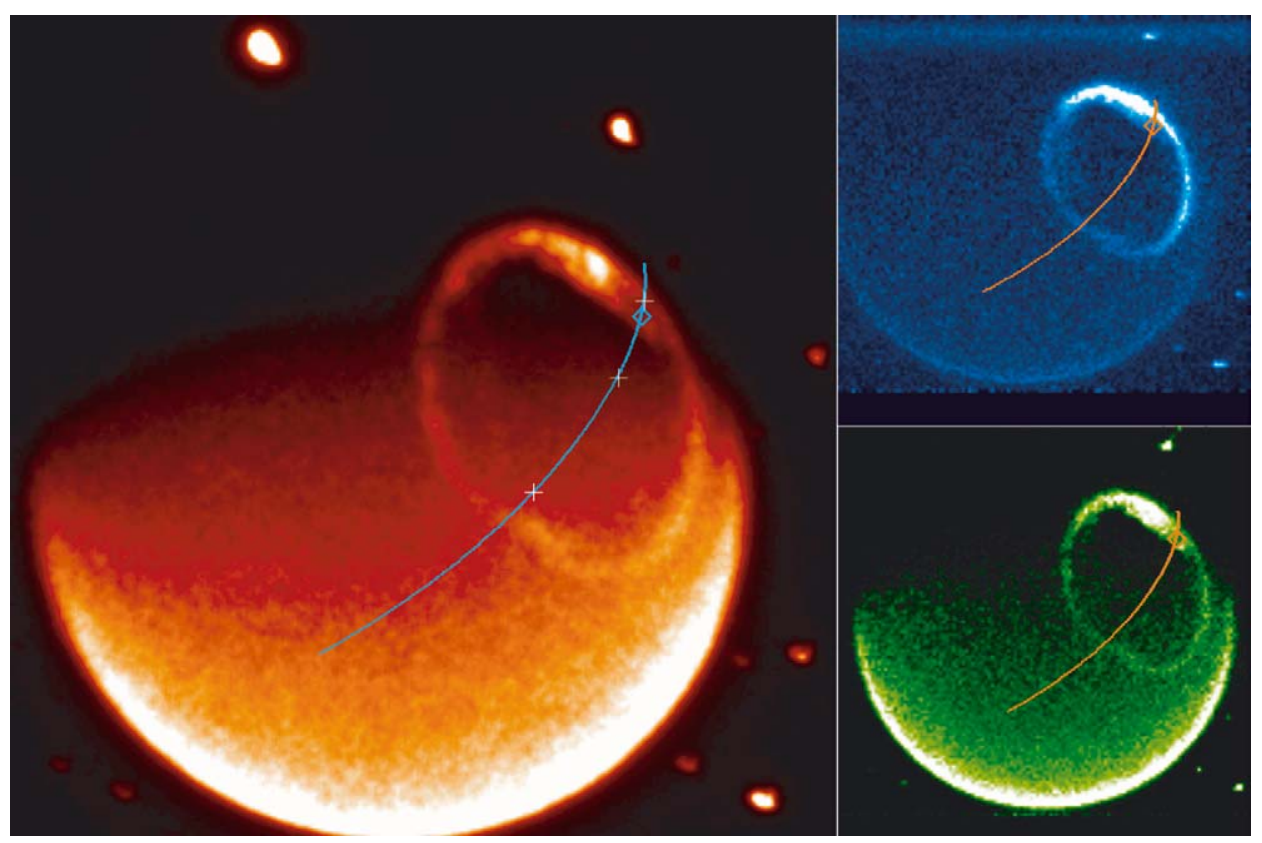

Figure 11. Three FUV images taken on June 24, 2000 at 06:22:20 UT. The WIC image is given in red, SI-12 in blue, and SI-13 in green. The ground-track of FAST is given as well as the location during image integration (diamond). Plus signs mark the location of FAST at 06:20, 06:30, and 06:40. Copyright 2001 American Geophysical Union. Reproduced by permission of American Geophysical Union from (Frey et al., 2001).

Now we want to go one step further and use the SI-13 results of this pass to estimate the energies and fluxes and compare them to the in-situ FAST measurements. We follow the scheme of section 8 and estimate a mean proton energy of $25 \mathrm{keV}$. This is a reasonable value for the nightside aurora shortly after a substorm and is also in agreement with FAST measurements. The proton contribution to the SI-13 signal is given in the top panel of Figure 15. The proton-corrected data from WIC and SI-13 were then used to estimate the mean electron energy in the middle panel of Figure 15. As this was a very active period with Kp values over 4 for more then 21 hours before the FAST measurements, we had to correct the ratios for the disturbed atmosphere. High geomagnetic activity causes the atmospheric oxygen to rise and produce more absorption of the SI-13 signal compared to quiet times. The reduced SI-13 signal creates a higher WIC/SI-13 ratio suggesting unreasonably high electron energies. A proper correction of the ratio values in Figure 7 leads to reasonable electron energies between 2 and $4 \mathrm{keV}$ in the nightside auroral oval. These energies are then applied to the SI-13 and WIC signals to estimate the energy flux and the result is shown in the lowest panel of Figure 15. The SI-13 values slightly overestimate the flux and the WIC values are 50\% larger. However, overall this result reflects the uncertainty we have to expect with this scheme of remote estimates. 


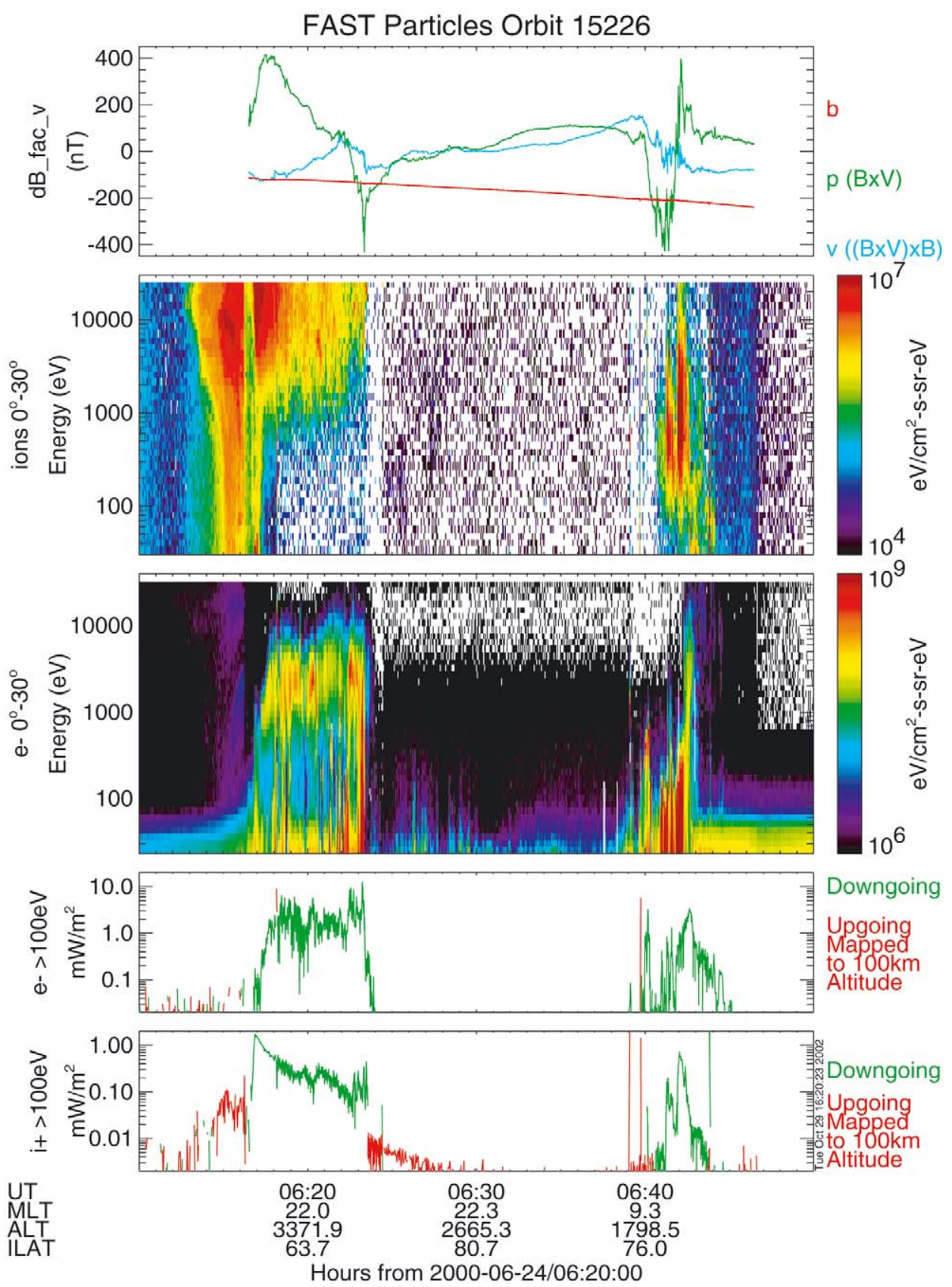

Figure 12. Observations by the FAST satellite during the pass through the images of FUV (Figure 11). The panels show the cross-track magnetic perturbation due to field-aligned currents, the differential ion and electron spectra in the loss cone and the integrated energy fluxes of electrons and ions. These data were the input to a full simulation of the expected signal in the FUV instrument. 


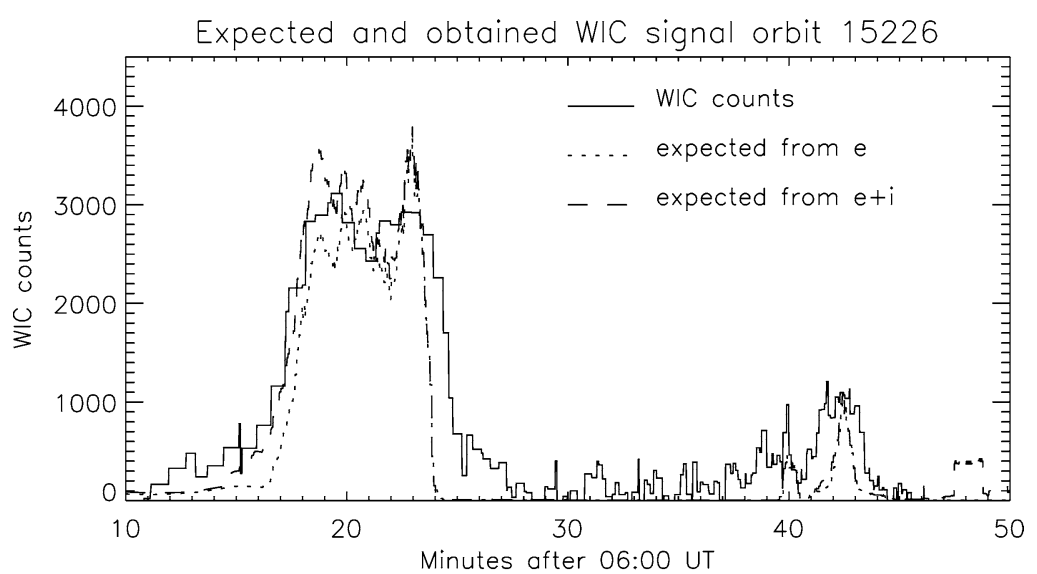

Figure 13. Comparison of the measured WIC signal along the FAST satellite track (solid line) and the expected signal from just precipitating electrons (dotted line) and the combination of electrons and protons (dashed line). Copyright 2001 American Geophysical Union. Reproduced by permission of American Geophysical Union from (Frey et al., 2001).

In addition to the already mentioned papers, some more studies were performed with NOAA satellite in-situ measurements together with WIC and SI-12 observations (Coumans et al., 2002). Many ion detectors on other spacecraft limit around $30 \mathrm{keV}$. Observations on the nightside show, that there may be substantial proton flux above $30 \mathrm{keV}$ (see also Figure 12) and that the total measured flux may miss an important part with higher energy. The MEPED detector on NOAA-satellites on the other hand, measures above $30 \mathrm{keV}$ and together with lower energy measurements the full precipitating spectrum can be characterized. In general, the WIC signal is well modeled if the different spatial resolutions of the sensors are properly accounted for. Most of the time the observed and the expected signal were close with local disagreements up to a factor of two. It was also shown, that the atmospheric composition influences the results to a lesser degree compared to the spatial and detector uncertainties. That study especially demonstrated that the missing high energy particle measurements $(>30 \mathrm{keV})$ in many other studies may explain some of the disagreement between measured and expected SI-12 signal (see for instance Figure 14).

Globally, ion precipitation accounts for about $10-17 \%$ of the integral energy precipitation into the aurora (Gérard et al., 2001). Locally however, this contribution can be much larger as demonstrated above with the FUV-FAST combination. In other studies it was found that in the dayside cusp region protons can carry about $30 \%$ of the total energy flux and sometimes may even dominate (Frey et al., 2002). Protons in the afternoon sector may carry more than $90 \%$ of the total energy flux (Hubert et al., 2001).

With the capability of IMAGE to observe the electron and proton produced aurora on a global scale, it is also possible to determine the global distribution of 


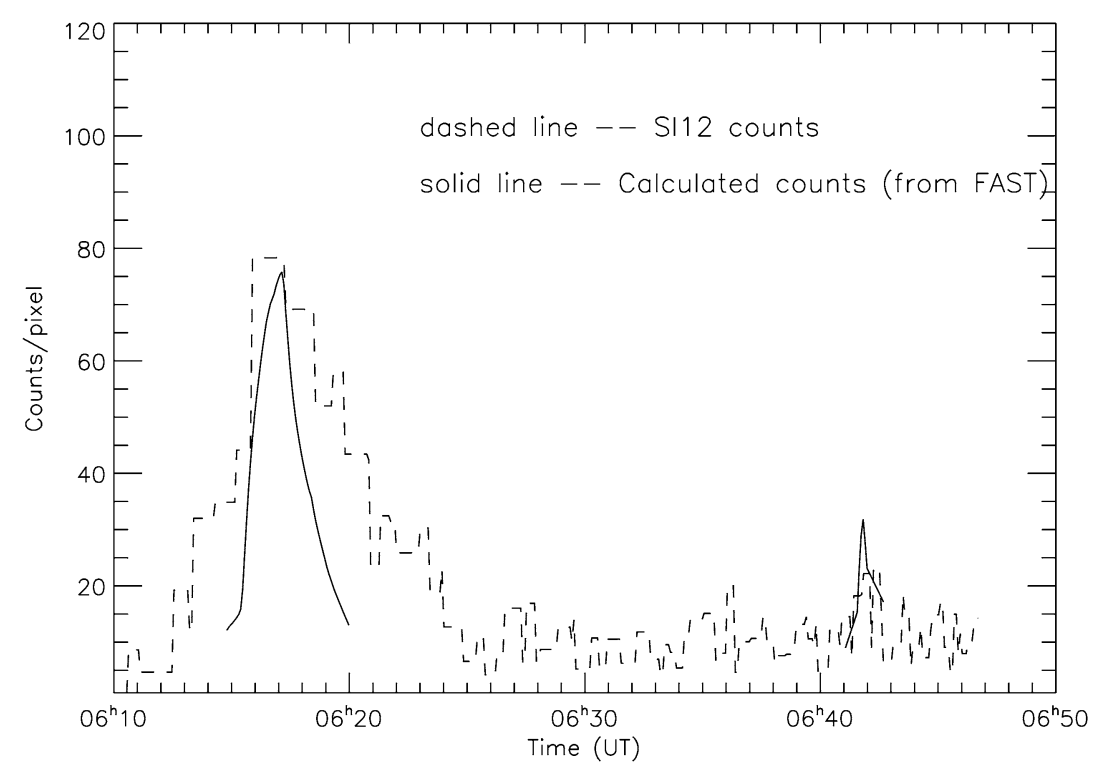

Figure 14. Comparison of the measured and expected SI-12 signal along the FAST satellite track. Copyright 2001 American Geophysical Union. Reproduced by permission of American Geophysical Union from (Gérard et al., 2001).

particle precipitation and energy input (Hubert et al., 2001; Hubert et al., 2002). It was shown that protons could contribute for more then $20 \%$ to the global energy input (hemispheric power) during quiet periods (Hubert et al., 2002). This approach goes one step further then the pure electron estimates from spacecraft like Polar (Chua et al., 2001). The lack of a proton detector forced them to account all aurora to electron excitation. As energetic protons act like low energy electrons, their estimates of electron energy should always be lower then the real electron energy, if substantial proton fluxes mix with electron precipitation.

Figure 16 illustrates an example of observations with IMAGE-FUV. The top row gives the three images in corrected instrument counts mapped to a geomagnetic grid. This is an example with substantial proton precipitation in the afternoon/evening region of 1700-2000 MLT. These energetic protons account for most of the WIC and SI-13 signal in that region. This is also shown in the bottom row, where the left image shows the mean electron energy from a pure electron assumption, compared to the middle panel where the proton contribution to the signal was removed before determination of the mean energy. The bottom right panel confirms that most of the electron energy flux occurs close to midnight and in the morning region, but not in the evening region.

(Hubert et al., 2002) used the quantitative calibration and method outlined above to determine the total hemispheric power input during quiet and disturbed times. Their results from global FUV observations were compared with the rather localized determinations by the NOAA satellites, and a reasonable agreement was 


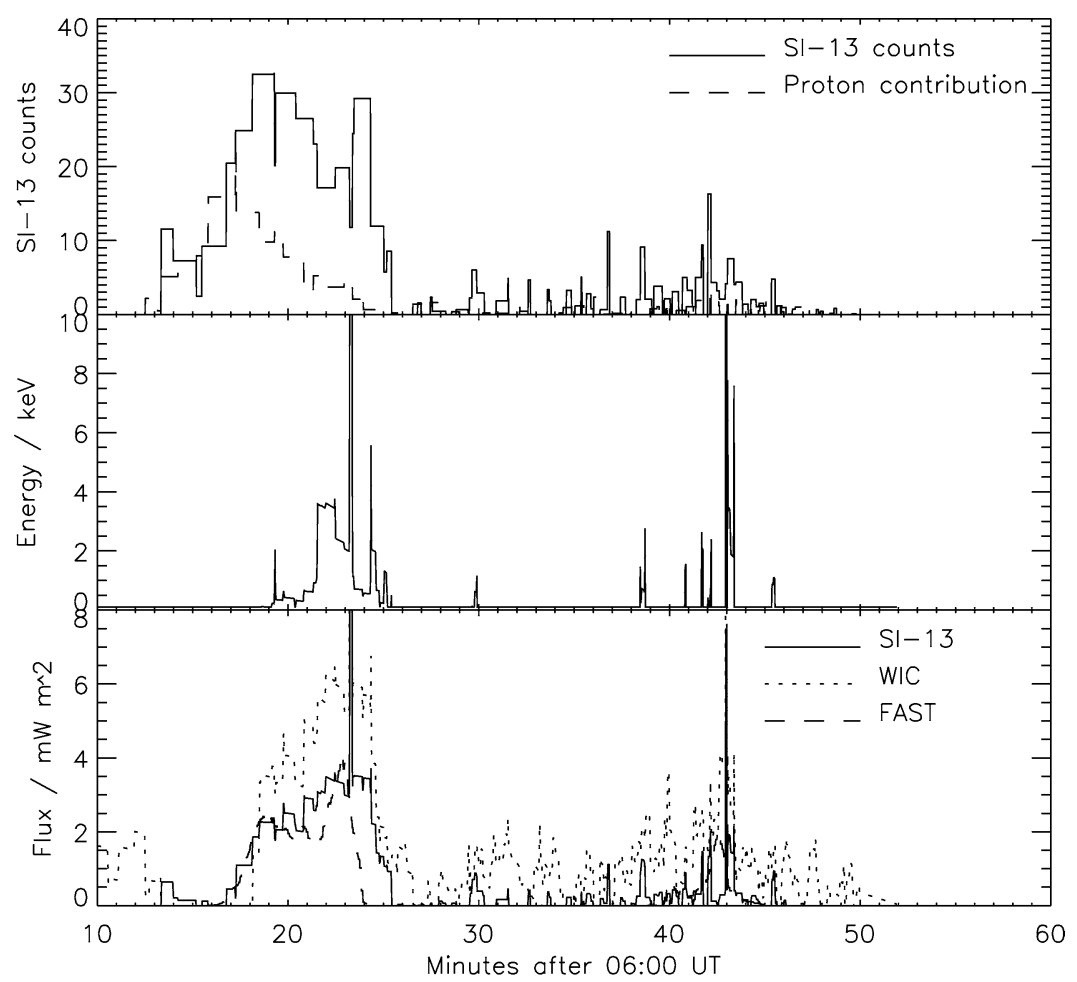

Figure 15. SI-13 counts along the track of FAST and the contribution to this signal by energetic protons (top panel). The middle panel shows the estimated mean energy of the precipitating electrons. The lowest panel shows the estimates of the precipitating electron energy flux from SI-13 (solid line), WIC (dotted line) and the FAST measurements (dashed line).

obtained. They were able to determine the temporal development of the auroral energy input carried by protons and electrons separately. The general agreement with the NOAA-satellite results confirmed the validity of the quantitative approach for the IMAGE-FUV analysis (Lummerzheim et al., 1997).

\section{Conclusions and Summary}

This paper describes the full way of obtaining quantitative results from IMAGEFUV observations. The whole process is based on a careful pre-launch instrument calibration, in-flight monitoring of the instrument response, the combination with model calculations of the expected auroral emissions, and validation with in-situ measurements by low-altitude spacecraft. The method as outlined here includes several simplifications like the description of particle fluxes by simple differential energy distributions (Maxwellian, kappa) and the constant atmospheric composition. Comparisons with satellite measurements also have to deal with the different spatial resolutions of the remote imager and the in-situ detectors. After all, reason- 


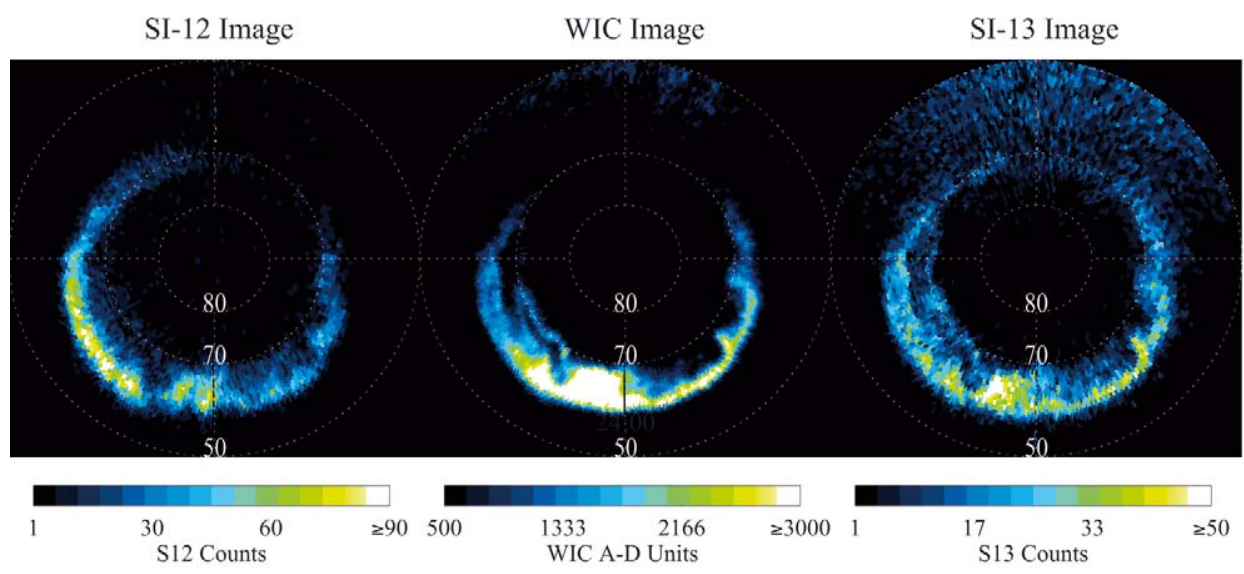

Uncorrected e- Mean Energy

Final e- Mean Energy

Final e- Energy Flux

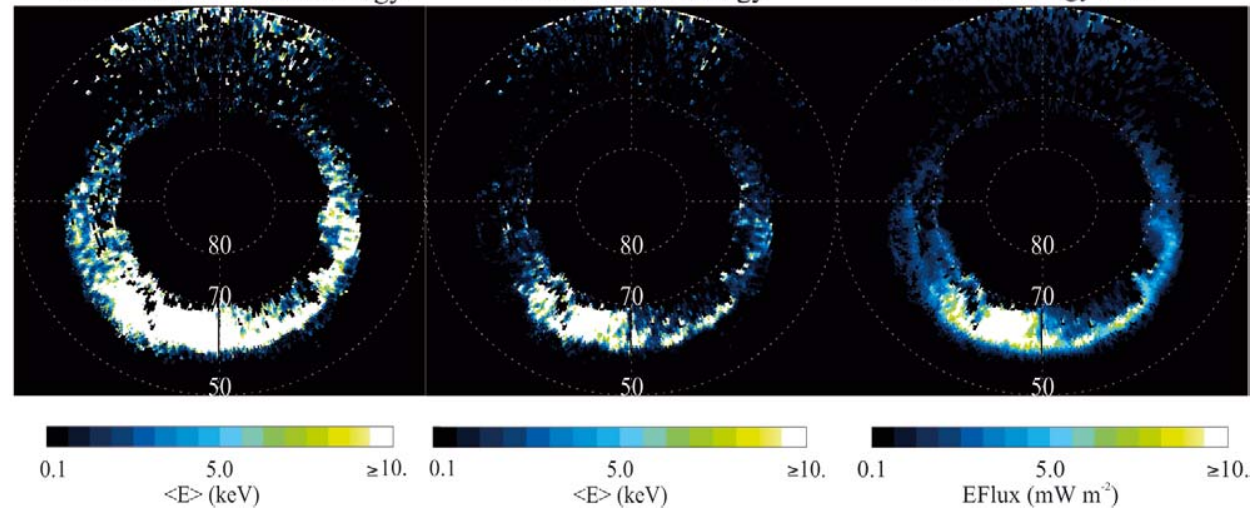

Figure 16. Full application of the quantitative analysis to a set of FUV images taken on October 28, 2000 at 11:38 UT. The top row shows the three images re-mapped into a geomagnetic grid with noon local time at the top and morning 0600 MLT to the right. Geomagnetic latitudes go down to 50 degrees in 10 degrees steps. The lower left image shows the mean electron energy derived from the image set, if all WIC and SI-13 signal is assumed to be produced by electrons. The middle bottom image shows the result after correction for the proton contribution. The lower right image shows the distribution of the energy flux carried by electrons.

able agreement was found in most cases as long as a factor of up to 2 difference between the measured and calculated results is accepted. The scheme of quantitative analysis of FUV observations has been used in several studies which so far had a large component of the validation of results. After the general validation has been achieved, more detailed investigations of auroral processes can be performed. Such studies can aim at the original goals of the IMAGE mission as e.g. the determination of the energy input from the solar wind into the ionosphere during quiet and disturbed times or the temporal development of the energy dissipation during all phases of the storm/substorm cycle. 


\section{Acknowledgements}

We are grateful to the many people whose dedication and hard work resulted in the IMAGE FUV data set, this includes the NASA, South West Research Institute, and the IMAGE-SMOC teams. The IMAGE FUV investigation was supported by NASA through SWRI subcontract number 83820 at the University of California at Berkeley under contract number NAS5-96020. J.-C.Gérard is supported by the Belgian National Fund for Scientific Research (FNRS). His work was funded by the PRODEX program of the European Space Agency (ESA) and the Belgian Fund for Collective Fundamental Research (grant FRFC 97-2.4569.97). We are indebted to the PI C.W. Carlson for the use of FAST data.

\section{References}

Ajello, J.M. and Shemansky, D.E.: 1985, 'A reexamination of important N2 cross sections by electron impact with application to the dayglow: The Lyman-Birge-Hopfield band system and N I (199.99 nm)', J. Geophys. Res. 90, 9845.

Anger, C.D., Babey, S.K., Broadfoot, A.L., Brown, R.G., Cogger, L.L., Gattinger, R., Haslett, J.W., King, R.A., McEwen, D.J., Murphree, J.S., Richardson, E.H., Sandel, B.R., Smith, K. and Jones, A.V.: 1987, 'An ultraviolet auroral imager for the Viking spacecraft', Geophys. Res. Lett. 14, 387.

Cassatella, A., Altamore, A., Gonzalez-Riestra, R., Ponz, J.D., Barbero, J., Talavera, A. and Wamsteker, W.: 2000, 'The INES system, 2, Ripple correction and absolute calibration for the IUE high resolution spectra', Astron. Astrophys. Suppl. 141, 331.

Chua, D, Parks, G., Brittnacher, M., Peria, W., Germany, G., Spann, J. and Carlson, C.: 2001, 'Energy characteristics of auroral electron precipitation: a comparison of substorms and pressure pulse related auroral activity', J. Geophys. Res. 106, 5945.

Coumans, V., Gérard, J.-C., Hubert, B. and Evans, D.S.: 2002, 'Electron and proton excitation of the FUV aurora: simultaneous IMAGE and NOAA observations', J. Geophys. Res. 107(A11), 1347, doi:10.1029/2001JA009233.

Drob, D.P., Meier, R.R., Picone, J.M., Strickland, D.J., Cox, R.J. and Nicholas, A.C.: 1999, 'Atomic oxygen in the thermosphere during the July 13, 1982, proton event deduced from far ultraviolet images', J. Geophys. Res. 104, 4267.

Frank, L.A., Craven, J.D., Ackerson, K.L., English, M.R., Eather, R.H. and Crovillano, R.L.: 1981, 'Global auroral imaging instrumentation for the Dynamics Explorer mission', Space Sci. Instrum. 5, 369-393.

Frank, L.A. and Craven, J.D.: 1988, Imaging results from Dynamics Explorer 1, Rev. Geophys. 2, 249.

Frey, H.U., Mende, S.B., Carlson, C.W., Gérard, J.-C., Hubert, B., Spann, J., Gladstone, R. and Immel, T.J.: 2001, 'The electron and proton aurora as seen by IMAGE-FUV and FAST', Geophys. Res. Lett. 28, 1135.

Frey, H.U., Mende, S.B., Immel, T.J., Fuselier, S.A., Claflin, E.S., Gérard, J.-C. and Hubert, B.: 2002, 'Proton aurora in the cusp', J. Geophys. Res. 107(A7), 1091, 10.1029/2001JA900161.

Gérard, J.-C., Hubert, B., Bisikalo, D.V. and Shematovich, V.I.: 2000, 'A model of the Lyman- $\alpha$ line profile in the proton aurora', J. Geophys. Res. 105, 15795.

Gérard, J.-C., Hubert, B., Meurant, M., Shematovich, V.I., Bisikalo, D.V., Frey, H., Mende, S., Gladstone, G.R. and Carlson, C.W.: 2001, 'Observation of the proton aurora with IMAGE FUV imager and simultaneous ion flux in situ measurements', J. Geophys. Res. 106, 28939.

Gladstone, G.R.: 1994, 'Simulations of DE-1 UV airglow images', J. Geophys. Res. 99, 11,441. 
Gladstone, R., Mende, S.B., Frey, H.U., Geller, S.P., Immel, T.J., Lampton, M., Spann, J., Gerard, J.-C., Habraken, S., Renotte, E., Jamar, C., Rochus, P. and Lauche, H.: 2000, 'Stellar Calibration of the WIC and SI Imagers and the GEO Photometers on IMAGE/FUV', Trans. AGU 81, 48, F1034.

Germany, G.A., Torr, M.R., Torr, D.G. and Richards, P.G.: 1994, 'Use of FUV auroral emissions as diagnostic indicators', J. Geophys. Res. 99, 383.

Germany, G.A., Parks, G.K., Brittnacher, M., Cumnock, J., Lummerzheim, D., Spann, J.F., Chen, L., Richards, P.G. and Rich, F.J.: 1997, 'Remote determination of auroral energy during substorm activity', Geophys. Res. Lett. 24, 995.

Hardy, D.A., Gussenhoven, M.S. and Brautigam, D.: 1989, 'A statistical model of auroral ion precipitation', J. Geophys. Res. 94, 370.

Hardy, D.A., McNeil, W., Gussenhoven, M.S. and Brautigam, D.: 1991, 'A statistical model of auroral ion precipitation: 2. Functional representation of the average patterns', J. Geophys. Res. 96, 5539.

Hedin, A.E.: 1991, 'Extension of the MSIS thermosphere model into the middle and lower atmosphere', J. Geophys. Res. 96, 1159.

Hubert, B., Gerard, J.-C., Bisikalo, D.V., Shematovich, V.I. and Solomon, S.C.: 2001, 'The role of proton precipitation in the excitation of auroral FUV emissions', J. Geophys. Res. 106, 21475.

Hubert, B., Gerard, J.-C., Evans, D.S., Meurant, M., Mende, S.B., Frey, H.U. and Immel, T.J.: 2002, 'Total electron and proton energy input during auroral substorms: remote sensing with IMAGEFUV', J. Geophys.Res. 107(A8), 10.1029/2001JA009229.

Hunten, D.M., Roach, F.E. and Chamberlain, J.W.: 1956, A photometric unit for the airglow and aurora, J. Atmos. Terr. Phys. 8, 345-346.

Immel, T.J., Craven, J.D. and Nicholas, A.C.: 2000, 'An empirical model of the OI FUV dayglow from DE-1 images', J. Atmos. Solar-Terr. Phys. 62, 47.

Lummerzheim, D., Brittnacher, M., Evans, D., Germany, G.A., Parks, G.K., Rees, M.H., and Spann, J.F.: 1997, 'High time resolution study of the hemispheric power carried by energetic electrons into the ionosphere during the May 19/20, 1996 auroral activity', Geophys. Res. Lett. 24, 987.

Lummerzheim, D., Galand, M., Semeter, J., Mendillo, M.J., Rees, M.H. and Rich, F.J.: 2001, 'Emission of O I (630 nm) in proton aurora', J. Geophys. Res. 106, 141.

Lummerzheim, D. and Galand, M.: 2001, 'The profile of the hydrogen $\mathrm{H}_{\beta}$-emission line in proton aurora', J. Geophys. Res. 106, 23.

Meier, R.R.: 1991, 'Ultraviolet spectroscopy and remote sensing of the upper atmosphere', Space Sci. Rev. 58, 1.

Mende, S.B., Heetderks, H., Frey, H.U., Lampton, M., Geller, S.P., Habraken, S., Renotte, E., Jamar, C., Rochus, P., Spann, J., Fuselier, S.A., Gerard, J.-C., Gladstone, R., Murphree, S. and Cogger, L.: 2000, 'Far ultraviolet imaging from the IMAGE spacecraft', Space Sci. Rev. 91, 243.

Murphree, J.S., King, R.A., Payne, T., Smith, K., Reid, D., Adema, J. and Gordon, B.: 1994, 'The Freja Ultraviolet Imager', Space Science Rev. 70, 421-446.

Solomon, S.C., Hays, P.B. and Abreu, V.: 1988, 'The auroral 6300 A emission: observation and modelling', J. Geophys. Res. 93, 9867.

Solomon, S.C.: 2001, 'Auroral particle transport using Monte Carlo hybrid methods', J. Geophys. Res. 106, 107.

Strickland, D.J. and Anderson, Jr., D.E.: 1983, 'Radiation transport effects on the OI 1356-AA limb intensity profile in the dayglow', J. Geophys. Res. 88, 9260.

Strickland, D.J., Jasperse, J.P. and Whalen, J.A.: 1983, 'Dependence of auroral FUV emissions on the incident electron spectrum and neutral atmosphere', J. Geophys. Res. 88, 8051.

Strickland, D.J., Daniell, Jr., R.E., Jasperse, J.R. and Basu, B.: 1993, 'Transport-theoretic model for the electron-proton-hydrogen atom aurora', J. Geophys. Res. 98, 21533.

Strickland, D.J., Cox, R.J., Meier, R.R. and Drob, D.P.: 1999, 'Global O/ $\mathrm{N}_{2}$ derived from DE-1 FUV dayglow data: Technique and examples from two storm periods', J. Geophys. Res., 104, 4251. 